\title{
Axioms for Euclidean Green's Functions II
}

\author{
Konrad Osterwalder ${ }^{\star}$ \\ Jefferson Laboratory of Physics, Harvard University, Cambridge, Massachusetts, USA \\ Robert Schrader \\ Institut für Theoretische Physik, Freie Universität Berlin, Berlin \\ with an Appendix by Stephen Summers
}

Received December 3, 1974; in revised form January 16, 1975

\begin{abstract}
We give new (necessary and) sufficient conditions for Euclidean Green's functions to have analytic continuations to a relativistic field theory. These results extend and correct a previous paper.
\end{abstract}

\section{Table of Contents}

I. Introduction . . . . . . . . . . . . . . . . . . . . . . . . . . . . . . . . . . 281

II. Notations . . . . . . . . . . . . . . . . . . . . . . . . . . . . . . . . . . . 283

III. The Equivalence Theorem Revisited . . . . . . . . . . . . . . . . . . . . . . . . . . 285

IV. The Main Result: Another Reconstruction Theorem . . . . . . . . . . . . . . . . . 287

IV.1 Linear Growth Condition and Statement of Results . . . . . . . . . . . . . . . . . 287

IV.2 Proof of Theorem $E^{\prime} \rightarrow R^{\prime}$. . . . . . . . . . . . . . . . . . . . . . . . . . 288

V. The Analytic Continuation . . . . . . . . . . . . . . . . . . . . . . . . . . . . 289

V.1 Real Analyticity . . . . . . . . . . . . . . . . . . . . . . . . . . . . . . . . . . . . . . 291

V.2 Towards the Real World . . . . . . . . . . . . . . . . . . . . . . . . . . . 293

VI. The Temperedness Estimate . . . . . . . . . . . . . . . . . . . . . . . . . . 297

VI.1 From Distributions to Functions . . . . . . . . . . . . . . . . . . . . . . . 297

VI.2 Continuing the Estimates . . . . . . . . . . . . . . . . . . . . . . . . . . 301

VII. Appendix . . . . . . . . . . . . . . . . . . . . . . . . . . . . . . . . . . 303

References . . . . . . . . . . . . . . . . . . . . . . . . . . . 305

\section{Introduction}

The passage to purely imaginary times has proven to be an extremely powerful tool both for the construction and for the discussion of relativistic quantum field theoretical models ${ }^{1}$. Obviously for such a procedure to make sense it is important to know how to go back again to real time.

In a previous paper "Axioms for Euclidean Green's functions" [12] (henceforth quoted as OS I) we claimed to have found necessary and sufficient conditions under which Euclidean Green's functions have analytic continuations whose boundary values define a unique set of Wightman distributions. These conditions

* Supported in part by the National Science Foundation under Grant MPS73-05037 A01.

Alfred P. Sloan Foundation Fellow.

1 For verification of this assertion the reader should consult the 1973 Erice Lectures on Constructive Quantum Field Theory [19], where also references and historical accounts can be found. 
were

(E0) Temperedness

(E1) Euclidean covariance

(E2) Positivity

(E3) Symmetry

(E4) Cluster property

As it turned out, a technical lemma (Lemma 8.8) in OS I is wrong (see Remark 2 below) and at present it is an open question whether the conditions $(E 0-E 4)$ as introduced in OS I are sufficient to guarantee the existence of a Wightman theory. They are certainly necessary. In this paper we give two different sets of sufficient conditions.

In Chapter III we replace the temperedness condition $(E 0)$ by a much stronger distribution condition $(\breve{E 0})$ and prove a new equivalence theorem: $(\breve{E 0}),(E 1-E 4)$ are necessary and sufficient conditions under which Euclidean Green's functions define a Wightman theory. Although E0 restores the equivalence theorem $E \leftrightarrow R$, this new condition is not suitable for application because it seems to be difficult to check. In Chapter IV we therefore introduce a condition $\left(E 0^{\prime}\right)$ which is only slightly different from the original $(E 0)$ : instead of simply assuming temperedness we now postulate that, roughly speaking, the order of the distributions $\mathfrak{S}_{n}$ (the Euclidean Green's functions) grows at most linearly in $n$, with bounds that grow no worse than $\alpha(n !)^{\beta}$ for arbitrary $\alpha$ and $\beta$. We call this the "linear growth condition". Assuming $\left(E 0^{\prime}\right),(E 1-E 4)$ we can again reconstruct a Wightman theory with Wightman distributions $\mathfrak{W}_{n}$ which also obey a linear growth condition $\left(R 0^{\prime}\right)$. The construction of the Wightman distributions requires two main steps: first we analytically continue the Euclidean Green's functions to complex times (Chapter V). Second we establish estimates for these analytic functions, which allow us to prove that their boundary values are tempered distributions: the Wightman distributions (Chapter VI). It is interesting to note that the analytic continuation alone can be done using the old temperedness condition (E0) together with covariance $(E 1)$ and positivity $(E 2)$. It is only because the analytic continuation of one particular Schwinger function $\mathfrak{S}_{n}$ involves infinitely many $\mathfrak{\Xi}_{k}$ that we need some control over the growth of the order of $\Xi_{k}$ to obtain the necessary estimates. Our linear growth condition seems to be quite reasonable. It certainly holds for all field theory models for which the Wightman axioms have been established so far and a recent result of Glimm and Jaffe [7] shows that it will also hold for a $\varphi_{4}^{4}$ model, provided it exists, and if the two point function is a distribution in $\mathscr{S}^{\prime}\left(\mathbb{R}^{8}\right)$.

Remarks. 1) The proof of Lemma 8.8 in OS I was first questioned by Simon [16]. Subsequently one of us (R.S.) found the following counter example: $F(x, y)$ $=\exp (-x y), x>0, y>0$ is the Laplace transform of a tempered distribution in each variable separately, but not jointly.

2) A preliminary report of the results of this paper was presented by one of us (K.O.) at the 1973 Erice Summer School on Constructive Quantum Field Theory, see [13], p. 71. It should be pointed out that condition $E 0^{\prime}$ in [13] was a linear growth condition on the difference variable Euclidean Green's functions. Condition $E 0^{\prime}$ of this paper refers to the Euclidean Green's functions directly; it is more general, more natural and certainly more convenient for applications than $E 0^{\prime}$ in [13]. 
3) The construction of the analytic continuation of Schwinger functions which satisfy $E 0, E 1$, and $E 2$ was found simultaneously by Glaser [6], who also eastablished the connection with his earlier work [5] on the interplay between positivity and analyticity. He too noted that in order to prove temperedness on the boundary of the analyticity domain an assumption stronger than $E 0$ seems to be necessary, but he remarked that $E 0-E 4$ lead to a modified Wightman theory with vacuum expectation values which are hyperfunctions but not necessarily tempered distributions.

4) Nelson's axioms [11] imply the Wightman axioms and hence by OS I also $E 0-E 4$. It is also easy to derive $E 0-E 4$ from Nelson's axioms directly; the crucial step is to prove positivity E2 using the Markoff and the reflection properties, see [19], p. 104. E0' seems to be related to Nelson's "scale condition", see Nelson [11]. On the other hand, to derive Nelson's axioms from $E 0^{\prime}, E 1-E 4$ one has to introduce additional assumptions, see Fröhlich [3] and Simon [15]. Nelson's axioms are more restrictive than $E 0^{\prime}, E 1-E 4$ and thus lead to a richer structure. On the other hand they seem to be harder to work with in constructive field theory: for none of the non-trivial models, constructed so far, has the Markoff property of Symanzik and Nelson (Relation (1) in [11]) been verified.

5) Though in this paper we deal with the theory of one real scalar field only, the results can be extended in an obvious way to theories with a denumerable number of arbitrary spinor fields, see Chapter 6 of OS I.

6) With the obvious changes the connections between subsets of the axioms for the Euclidean Green's functions and subsets of the Wightman axioms are as discussed in OS I.

7) Constantinescu and Thalheimer have extended the scheme of axioms $E 0 / E 0^{\prime}, E 1-E 4$ to Jaffe fields [1].

Acknowledgements. We thank Prof. A. Jaffe and Prof. K. Pohlmeyer for helpful discussions and Prof. V. Glaser for sending us a copy of his paper prior to publication. We also thank Prof. G.-F. Dell'Antonio for his warm hospitality at the Università di Napoli, where part of this work was done.

\section{Notations}

In this section we introduce some (partially new) notation and restate a few technical results from OS I.

Unless stated otherwise, $x$ denotes a point in $\mathbb{R}^{4}$ with coordinates $\left(x^{0}, x^{1}, x^{2}, x^{3}\right)$ $\equiv\left(x^{0}, \vec{x}\right)$. A point in $\mathbb{R}^{4 n}$ will be written as

$$
\underline{x}=\left(x_{1}, \ldots x_{n}\right), \quad x_{i} \in \mathbb{R}^{4} .
$$

For integrals we write $\int \ldots d^{4 n} x$ or simply $\int \ldots d x$.

We will use the following open sets

$$
\begin{aligned}
\mathbb{R}_{+}^{4} & =\left\{x \in \mathbb{R}^{4} \mid x^{0}>0\right\} \\
\mathbb{R}_{+}^{4 n} & =\left\{\underline{x} \in \mathbb{R}^{4 n} \mid x_{j}^{0}>0 \text { for all } j=1, \ldots n\right\} \\
\mathbb{R}_{<}^{4 n} & =\left\{\underline{x} \in \mathbb{R}^{4 n} \mid x_{j+1}^{0}>x_{j}^{0} \text { for all } j=1, \ldots n-1\right\} \\
\mathbb{R}_{0}^{4 n} & =\left\{\underline{x} \in \mathbb{R}^{4 n} \mid x_{i} \neq x_{j} \text { for all } 1 \leqq i<j \leqq n\right\} \\
\mathbb{C}_{+} & =\{z \in \mathbb{C} \mid \operatorname{Re} z>0\} \\
\mathbb{C}_{+}^{n} & =\left\{\left(z_{1}, \ldots z_{n}\right) \mid z_{j} \in \mathbb{C}_{+} \text {for all } j=1, \ldots n\right\} .
\end{aligned}
$$


On the Schwartz space $\mathscr{S}\left(\mathbb{R}^{m}\right)$ we will work with the following norms

$$
|f|_{p}=\sup _{\substack{x \in \mathbb{R}^{m} \\|\underline{\mid}| \leqq p}}\left|\left(1+x^{2}\right)^{p / 2}\left(D^{\underline{\alpha}} f\right)(x)\right|,
$$

where $p \in \mathbb{Z}_{+}=\{1,2, \ldots\}, f \in \mathscr{S}\left(\mathbb{R}^{m}\right)$. We use the standard multiindex notation: $\underline{\alpha}=\left(\alpha_{1}, \ldots \alpha_{m}\right) ;|\underline{\alpha}|=\sum_{1}^{m} \alpha_{i}, D^{\underline{\alpha}}=\prod_{1}^{m}\left(\frac{\partial}{\partial x_{i}}\right)^{\alpha_{i}} ; x^{2}=\sum_{1}^{m}\left(x_{i}\right)^{2}$.

By $\mathscr{S}_{0}\left(\mathbb{R}^{4 n}\right)$ we denote the topological subspace of $\mathscr{S}\left(\mathbb{R}^{4 n}\right)$ of all those functions which together with all their derivatives vanish on the complement $\sim \mathbb{R}_{0}^{4 n}$ of $\mathbb{R}_{0}^{4 n}$.

As in OS I we denote by $\mathfrak{S}_{n}(\underline{x})$ the Euclidean Green's functions and by $\mathfrak{B}_{n}(\underline{x})$ the Wightman distributions. The "difference variable" Euclidean Green's functions $S_{n-1}(\underline{\xi})$ and Wightman distributions $W_{n-1}(\underline{\xi})$ are formally defined by

$$
\begin{aligned}
\mathfrak{S}_{n}(\underline{x}) & =S_{n-1}(\underline{\xi}) \\
\mathfrak{W}_{n}(\underline{x}) & \left.=W_{n-1} \underline{\xi}\right)
\end{aligned}
$$

respectively, where $\xi_{k}=x_{k+1}-x_{k}, k=1, \ldots n-1$. The Wightman axioms will be labelled as follows: $(R 0)$ Distribution property, $(R 1)$ Relativistic invariance, $(R 2)$ Positivity, $(R 3)$ Local commutativity, $(R 4)$ Cluster property, and $(R 5)$ Spectral condition.

The remainder of this section will be needed in Chapter III only.

For $O$ an open set in $\mathbb{R}^{m}, \mathscr{S}(O)$ denotes the subspace of $\mathscr{S}\left(\mathbb{R}^{m}\right)$ of functions with support in $\bar{O}$, given the induced topology. The dual space of the topological quotient space $\mathscr{S}\left(\mathbb{R}^{m}\right) / \mathscr{S}(O)$ is the polar of $\mathscr{S}(O)$, which is the set of all tempered distributions with support in $\sim O$. By $\mathscr{S}(\bar{O})$ we denote the set of $C^{\infty}$ functions on $O$ which decrease strongly with all their derivatives as $|x| \rightarrow \infty$ in $O$ and whose derivatives all have a continuous extension to the closure $\bar{O}$ of $O$. On $\mathscr{S}(\bar{O})$ we define a topology by the norms

$$
|g|_{p, O}=\sup _{\substack{x \in O \\|\underline{\alpha}| \leqq p}}\left|\left(1+x^{2}\right)^{p / 2}\left(D^{\underline{\alpha}} g\right)(x)\right| .
$$

$\mathscr{S}(\bar{O})$ is of course not a subspace of $\mathscr{S}\left(\mathbb{R}^{m}\right)$, but as the following lemma shows, an element in $\mathscr{S}(\bar{O})$ can always be regarded as the restriction to $\bar{O}$ of an element in $\mathscr{S}\left(\mathbb{R}^{m}\right)$.

Lemma 2.1. Let $O$ be an open set in $\mathbb{R}^{m}$. Then $\mathscr{S}(\bar{O})$ is isomorphic to $\mathscr{S}\left(\mathbb{R}^{m}\right) / \mathscr{S}(\sim \bar{O})$.

This lemma follows from the fact that the set of functions $f_{+}$in $\mathscr{S}(\bar{O})$ which are restrictions to $\bar{O}$ of functions $f \in \mathscr{S}\left(\mathbb{R}^{m}\right)$ is dense in $\mathscr{S}(\bar{O})$ and from Whitney's extension theorem, see Whitney [21], Hörmander [9], Vladimirov [20] and also Lemma 8.1 in OS I. From Whitney's extension theorem it follows immediately that the norms

$$
\|g\|_{p, o}=\inf _{h \in \mathscr{S}(\sim \bar{o})}|g+h|_{p}
$$

are equivalent to the norms defined by (2.2), $\left(g \in \mathscr{S}\left(\mathbb{R}^{m}\right)\right)$. In particular, for $O=V_{+}^{n} \equiv\left\{\underline{x} \mid x_{i}^{0}>0\right.$ and $\left(x_{i}^{0}\right)^{2}>\vec{x}_{i}^{2}$, all $\left.i=1, \ldots n\right\}$, we have the following 
Lemma 2.2. Suppose $f_{+} \in \mathscr{S}\left(\overline{V_{+}^{n}}\right)$ and $p \in \mathbb{Z}_{+}$. Then there exists a function $g \in \mathscr{S}^{(p)}\left(\mathbb{R}^{4 n}\right)$ such that $g(\underline{x})=f_{+}(\underline{x})$ for $\underline{x} \in \overline{V_{+}^{n}}$ and

$$
\left|f_{+}\right|_{p, V_{+}^{n}} \leqq\|g\|_{p, V_{+}^{n}} \leqq \gamma\left|f_{+}\right|_{2 p, V_{+}^{n}}
$$

with $\gamma=\gamma(n, p)=\left[c 2^{n}(p+1)\right]^{2 p+1}$ for some constant $c$ independent of $n$ and $p$.

Here $\mathscr{S}^{(p)}\left(\mathbb{R}^{4 n}\right)$ is the closure of $\mathscr{S}\left(\mathbb{R}^{4 n}\right)$ in the topology defined by the $|\cdot|_{p^{-}}$ norm. Notice that the first inequality in (2.4) is trivial. The second one is a sharp form of Whitney's extension theorem and follows from a detailed analysis of the proof given in [9]. We omit the details.

As an easy consequence of Lemmas 2.1 and 2.2 we get

Lemma 2.3. Let $\hat{W}$ be a distribution in $\mathscr{S}^{\prime}\left(\mathbb{R}^{4 n}\right)$ with support in $\overline{V_{+}^{n}}$ such that for all $f \in \mathscr{S}\left(\mathbb{R}^{4 n}\right)$

$$
|\hat{W}(f)| \leqq w|f|_{p} .
$$

Then $\hat{W}$ also defines a distribution in $\mathscr{S}^{\prime}\left(\overline{V_{+}^{n}}\right)$, again denoted by $\hat{W}$, such that for all $f_{+} \in \mathscr{S}\left(\overline{V_{+}^{n}}\right)$

$$
\left|\hat{W}\left(f_{+}\right)\right| \leqq w \cdot \gamma\left|f_{+}\right|_{2 p, \overline{V_{+}^{n}}},
$$

with $\gamma$ as in Lemma 2.2 .

For $f \in \mathscr{S}\left(\mathbb{R}_{+}^{4 n}\right)$ and $q \in \overline{\mathbb{R}_{+}^{4 n}}$ we define

$$
\check{f}(q)=\int \exp \left[-\sum_{j=1}^{n}\left(q_{j}^{0} x_{j}^{0}-i \vec{q}_{j} \vec{x}_{j}\right)\right] f(\underline{x}) d^{4 n} x .
$$

The following lemma follows immediately from Lemma 8.2 in OS I.

Lemma 2.4. The map $f \rightarrow \breve{f}$ defined by (2.6) is a continuous map from $\mathscr{S}\left(\mathbb{R}_{+}^{4 n}\right)$ to $\mathscr{S}\left(\overline{\mathbb{R}_{+}^{4 n}}\right)$ with dense range and trivial kernel.

Now we define $\check{\mathscr{S}}\left(\mathbb{R}_{+}^{4 n}\right)$ to be the linear space $\mathscr{S}\left(\mathbb{R}_{+}^{4 n}\right)$ equipped with the topology given by the family of seminorms

$$
|f|_{p}=|\check{f}|_{p, \overline{\mathbb{R}^{4}}}, \quad p=1,2, \ldots .
$$

Note that $\check{\mathscr{S}}\left(\mathbb{R}_{+}^{4 n}\right)$ is not complete. By Lemma 2.4 the topology of $\check{\mathscr{S}}\left(\mathbb{R}_{+}^{4 n}\right)$ is weaker than the original topology of $\mathscr{S}\left(\mathbb{R}_{+}^{4 n}\right)$ and hence

$$
\check{\mathscr{S}}^{\prime}\left(\mathbb{R}_{+}^{4 n}\right) \subset \mathscr{S}^{\prime}\left(\mathbb{R}_{+}^{4 n}\right) \text {. }
$$

\section{The Equivalence Theorem Revisited}

In this section we introduce a new distribution property $(\breve{E 0})$ for the Euclidean Green's functions and prove that $\breve{E 0}$ together with $E 1-E 4$ is equivalent to the usual Wightman axioms. The new condition is as follows

$$
\left\{\begin{array}{lll}
\text { and } & \mathfrak{S}_{n} \in \mathscr{S}_{0}^{\prime}\left(\mathbb{R}^{4 n}\right), & \mathfrak{S}_{0}=1 \\
S_{n-1} \in \check{\mathscr{S}}^{\prime}\left(\mathbb{R}_{+}^{4(n-1)}\right), & n=1,2, \ldots .
\end{array}\right.
$$

Theorem $\mathbf{E} \leftrightarrow \mathbf{R}$ (revisited). The conditions E0, E1-E4 for the Euclidean Green's functions are equivalent to the Wightman axioms $R 0-R 5$ for the Wightman distributions. 
Although $\check{E 0}$ restores the equivalence theorem $E \leftrightarrow R$, this new condition is not very satisfactory from the point of view of applications. In praxi the continuity of $S_{n-1}$ with respect to the $|\cdot|_{p}$-norms is a condition which seems to be difficult to check; as we shall see below, $E 0$ immediately implies that $S_{n-1}$ is the FourierLaplace transform of a distribution $\hat{W}_{n-1}$ that has the desired support properties. From the point of view of constructive quantum field theory the results of the next section will be the crucial ones.

We now turn to the proof of Theorem $E \leftrightarrow R$. The derivation of $\check{E 0}, E 1-E 4$ from the Wightman axioms follows the arguments of OS I; all that remains to be verified is the additional condition $S_{n-1} \in \check{\mathscr{S}}^{\prime}\left(\mathbb{R}_{+}^{4(n-1)}\right)$. As in OS I, Chapter 5, we show that for $f \in \mathscr{S}\left(\mathbb{R}_{+}^{4 n}\right)$,

$$
S_{n-1}(f)=\hat{W}_{n-1}(\check{f}),
$$

where $\hat{W}_{n-1}$ is the Fourier transform of $W_{n-1}$, interpreted as a distribution in $\mathscr{S}^{\prime}\left(\overline{\mathbb{R}_{+}^{4 n}}\right)$, see also Lemma 2.1. This implies that for some $p$,

$$
\left|S_{n-1}(f)\right|<|f|_{p}^{2}
$$

and hence $S_{n-1}$ is an element in $\check{\mathscr{S}}^{\prime}\left(\mathbb{R}_{+}^{4(n-1)}\right)$.

Let us give an alternative and simple proof of $(3.1) /(3.2)$. For $\underline{\xi} \in \mathbb{R}_{+}^{4(n-1)}$, the function

$$
h_{\underline{\xi}}(q)=\exp \left[-\sum_{j=1}^{n-1}\left(\xi_{j} q_{j}-i \vec{\xi}_{j} \vec{q}_{j}\right)\right]
$$

is an element of $\mathscr{S}\left(\overline{V_{+}^{n-1}}\right)$, depending continuously on $\underline{\xi}$. Thus by Lemma 2.3 we may write $S_{n-1}(\underline{\xi})$ as

$$
S_{n-1}(\underline{\xi})=\hat{W}_{n-1}\left(h_{\underline{\xi}}\right) \text {. }
$$

Then for $f \in \mathscr{S}\left(\mathbb{R}_{+}^{4(n-1)}\right)$ with compact support we define

$$
\begin{aligned}
S_{n-1}(f) & =\int S_{n-1}(\underline{\xi}) f(\underline{\xi}) d^{4(n-1)} \xi \\
& =\int \hat{W}_{n-1}\left(h_{\underline{\xi}}\right) f(\underline{\xi}) d^{4(n-1)} \xi,
\end{aligned}
$$

the right hand side of (3.4) being taken as an ordinary Riemann integral. We now claim that for such $f$

$$
\int \hat{W}_{n-1}\left(h_{\underline{\xi}}\right) f(\underline{\xi}) d^{4(n-1)} \xi=\hat{W}_{n-1}(\check{f}),
$$

which proves (3.1) for a dense set in $\mathscr{S}\left(\mathbb{R}_{+}^{4(n-1)}\right)$ by continuity. For a proof of (3.5) we write $\breve{f}$ as

$$
\check{f}(\underline{q})=\int h_{\underline{\xi}}(q) f(\underline{\xi}) d^{4(n-1)} \xi \in \mathscr{S}\left(\overline{V_{+}^{n}}\right)
$$

and approximate it in $\mathscr{S}\left(\overline{V_{+}^{n}}\right)$ by Riemann sums.

Now we show how to modify the proof of $E \rightarrow R$. Starting from $S_{n-1} \in \check{\mathscr{S}}^{\prime}\left(\mathbb{R}_{+}^{4 n}\right)$ we define $\hat{W}_{n-1}$ by (3.1). This defines $\hat{W}_{n-1}$ on a dense set of $\mathscr{S}\left(\overline{\mathbb{R}_{+}^{4 n}}\right)$ - see Lemma 2.4 - and by assumption $\check{E 0}, \hat{W}_{n-1}$ is continuous with respect to the topology of $\mathscr{S}\left(\overline{\mathbb{R}_{+}^{4 n}}\right)$. Hence $W_{n-1}$ has a unique extension to a distribution in $\mathscr{S}^{\prime}\left(\overline{\mathbb{R}_{+}^{4 n}}\right)$. The proof of the remaining Wightman axioms now proceeds as in OS I. Equations (3.3)/(3.4) are easily verified, which shows that the $S_{n}$ are indeed the Euclidean Green's functions of the Wightman theory thus obtained. 


\section{The Main Result: Another Reconstruction Theorem}

\section{IV.1. Linear Growth Condition and Statement of Results}

As we have shown in the last chapter the equivalence $E \leftrightarrow R$ can be established

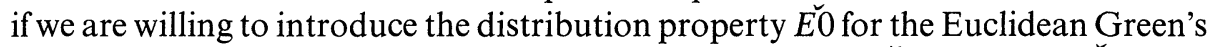
functions. In this chapter we show that we can avoid $E \overline{E 0}$ and the $|\cdot|$-norms altogether.

A sequence $\left\{\sigma_{n}\right\}_{n \in \mathbb{Z}_{+}}$of positive numbers is said to be of factorial growth if there exist constants $\alpha$ and $\beta$ such that

for all $n \in \mathbb{Z}_{+}$.

$$
\sigma_{n} \leqq \alpha(n !)^{\beta}
$$

We now define what we call the linear growth condition for the Euclidean Green's functions.

$$
\left(E 0^{\prime}\right)\left\{\begin{array}{l}
\Im_{0} \equiv 1, \Im_{n} \in \mathscr{S}_{0}^{\prime}\left(\mathbb{R}^{4 n}\right) \text { and there exist } s \in \mathbb{Z}_{+} \text {and a sequence } \\
\left\{\sigma_{n}\right\} \text { of factorial growth, such that } \\
\qquad\left|\Im_{n}(f)\right| \leqq \sigma_{n}|f|_{n \cdot s} \\
\text { for all } n \in \mathbb{Z}_{+}, f \in \mathscr{S}_{0}\left(\mathbb{R}^{4 n}\right) .
\end{array}\right.
$$

The following condition is slightly stronger than $E 0^{\prime}$.

$$
\left(E 0^{\prime \prime}\right)\left\{\begin{array}{l}
\Im_{0} \equiv 1, \Im_{n} \in \mathscr{S}^{\prime}\left(\mathbb{R}^{4 n}\right) \text { for all } n \in \mathbb{Z}_{+}, \text {and there exist } s \in \mathbb{Z}_{+} \text {and a } \\
\text { sequence }\left\{\sigma_{n}\right\} \text { of factorial growth such that } \\
\qquad\left|\Im_{n}\left(f_{1} \times f_{2} \cdots \times f_{n}\right)\right| \leqq \sigma_{n} \prod_{i=1}^{n}\left|f_{i}\right|_{s} \\
\text { for all } n \in \mathbb{Z}_{+}, f_{i} \in \mathscr{S}\left(\mathbb{R}^{4}\right) \text {. }
\end{array}\right.
$$

The following theorem contains the main result of this paper.

Theorem $E^{\prime}$ (or $\left.E^{\prime \prime}\right) \rightarrow R^{\prime}$. a) $A$ sequence of distributions $\left\{\mathbb{\Xi}_{n}\right\}_{n=0}^{\infty}$ satisfying $E 0^{\prime}$ (or E $0^{\prime \prime}$ ) and E1-E4 is the sequence of Euclidean Green's functions of a uniquely determined Wightman quantum field theory.

b) The Wightman distributions $\left\{\mathfrak{W}_{n}\right\}$ of that theory satisfy all the Wightman axioms $R 0-R 5$ and in addition

$$
\left(R 0^{\prime}\right)\left\{\begin{array}{l}
\text { there exists } w \in \mathbb{Z}_{+} \text {and a sequence }\left\{\omega_{n}\right\} \text { with } 0<\omega_{n} \leqq \alpha \beta^{n^{2}} \\
\text { for some constants } \alpha, \beta \text { and all } n \in \mathbb{Z}_{+} \text {, such that } \\
\qquad\left|\mathfrak{W}_{n}(f)\right| \leqq \omega_{n}|f|_{n \cdot w} \\
\text { for all } f \in \mathscr{S}\left(\mathbb{R}^{4 n}\right) \text {. }
\end{array}\right.
$$

Remarks. 1. As $E 0^{\prime \prime}$ implies $E 0^{\prime}$ (see Appendix) it is sufficient to prove $E^{\prime} \rightarrow R^{\prime}$. It is however worth noticing that a direct proof of $E^{\prime \prime} \rightarrow R^{\prime}$ would be much simpler that the proof of $E^{\prime} \rightarrow R^{\prime}$ presented in this paper. This will be explained in the introduction to Chapter V. Clearly, $E 0^{\prime}$ does not imply $E 0^{\prime \prime}$. Superficially speaking, while $E 0^{\prime \prime}$ requires $\mathfrak{\Xi}_{n}\left(x_{1} \ldots x_{n}\right)$ not to grow faster than $\prod_{i}\left(1+x_{i}^{2}\right)^{s / 2}$ for large values of the arguments, $E 0^{\prime}$ allows for a growth of the order of $\left(1+\sum_{i} x_{i}^{2}\right)^{n s / 2}$, and similarly for the singularities of $\mathfrak{S}_{n}$ at points of coinciding arguments. 
2. In constructive quantum field theory $E 0^{\prime \prime}$ holds for all models for which $E 0-E 4$ has been verified so far, see Glimm, Jaffe, and Spencer [8] remark below Theorem 1.1.8, and Fröhlich [3]. It is also reasonable to expect that $E 0^{\prime \prime}$ holds for models that live in the real world with three spatial dimensions: a recent result of Glimm and Jaffe [7] shows that in a $\left(\varphi^{4}\right)_{4}$ model $E 0^{\prime \prime}$ would follow essentially from the fact that $\widetilde{\Xi}_{2}$ is an element of $\mathscr{S}^{\prime}\left(\mathbb{R}^{8}\right)$.

3. Our methods do not allow for a factorial growth estimate on $\omega_{n}$ in $R 0^{\prime}$. On the other hand, assuming $R 0^{\prime}, R 1-R 5$ and $\omega_{n}$ of factorial growth, we can derive $E 0^{\prime}, E 1-E 4$; but the bounds we obtain for $\sigma_{n}$ are of the form $\alpha \beta^{n^{2}}$. This follows easily by first applying (2.4) on $h_{\xi}$ in Relation (3.4) and then using arguments of OS I with a sharpened version of Lemma 2.2 in OS I. Were it not for the obstacles of establishing the factorial growth bounds on $\sigma_{n}$ and $\omega_{n}$ respectively, one could again prove an equivalence theorem $E^{\prime} \leftrightarrow R^{\prime}$.

\section{IV.2. Proof of Theorem $E^{\prime} \rightarrow R^{\prime}$}

In this section we explain how to reconstruct the Wightman distributions from a given set of Euclidean Green's functions satisfying $E 0^{\prime}, E 1$ (Euclidean invariance) and $E 2$ (positivity) and verify the distribution property $R 0^{\prime}$. The remaining Wightman axioms can be established as in Sections 4.2-4.5 of OS I. The proofs of the theorems stated below will be given in subsequent chapters.

The existence of the Wightman distributions follows from an inductive construction of the analytic continuation of the Euclidean Green's functions and from bounds on these analytic continuations which are established inductively too. We always assume that we are given a sequence of Euclidean Green's functions $\left\{\Xi_{n}\right\}$ satisfying $E 0^{\prime}, E 1$ and $E 2$. By $S_{n-1}$ we denote the difference variable Green's functions. The initial step in our inductive procedure is to prove the following theorem.

Theorem 4.1. $\left(A_{0}\right)$ Real Analyticity: There are functions $S_{k}(\underline{\zeta})=S_{k}(\underline{\xi}+i \underline{\eta})$ analytic in some complex neighborhood of $\mathbb{R}_{+}^{4 k}$ such that for all $f \in \mathscr{S}\left(\mathbb{R}_{+}^{4 k}\right)$

$$
S_{k}(f)=\int S_{k}(\underline{\xi}) f(\underline{\xi}) d^{4 k} \xi .
$$

$\left(T E_{0}\right)$ Temperedness Estimate: The functions $S_{k}(\underline{\xi})$ satisfy

$$
\left|S_{k}(\underline{\xi})\right| \leqq \tau_{k}\left[\left(1+\max _{1 \leqq j \leqq k}\left|\vec{\xi}_{j}\right|\right)\left(1+\sum_{j=1}^{k} \xi_{j}^{0}\right)\left(1+\sum_{j=1}^{k}\left(\xi_{j}^{0}\right)^{-1}\right)\right]^{k t}
$$

for some sequence $\tau_{k}$ of factorial growth, some positive integer $t$ (not depending on $k$ ) and all $k \in \mathbb{Z}_{+}$, all $\underline{\xi} \in \mathbb{R}_{+}^{4 k}$.

In the $r$ 'th step of the induction we construct open subsets $C_{k}^{(r)}$ of $\mathbb{C}_{+}^{k}$ and prove the following theorem.

Theorem 4.2. $\left(A_{r}\right) \quad$ For fixed $\vec{\xi} \equiv\left(\vec{\xi}_{1}, \ldots \vec{\xi}_{k}\right)$ the functions $S_{k}\left(\underline{\xi^{0}} \mid \underline{\vec{\xi}}\right) \equiv S_{k}(\underline{\xi})$ have an analytic continuation $S_{k}\left(\underline{\zeta}^{0} \mid \underline{\vec{\xi}}\right)$ to the region $\underline{\zeta}^{0}=\underline{\xi}^{0}+i \underline{\eta}^{0} \in C_{k}^{(r)}$. $S_{k}\left(\underline{\zeta^{0}} \mid \underline{\xi}\right)$ is continuous in the variables $\vec{\xi}$.

$\left(T E_{r}\right) \quad$ For $\underline{\zeta}^{0} \in C_{k}^{(r)}$ and $\underline{\xi} \in \mathbb{R}^{3 k}$ the functions $S_{k}\left(\underline{\zeta}^{0} \mid \underline{\xi}\right)$ satisfy

$$
\left|S_{k}\left(\zeta^{0} \mid \underline{\xi}\right)\right| \leqq c_{k}\left[\left(1+\max _{j}\left|\vec{\xi}_{j}\right|\right)\left(1+\sum_{j}\left|\zeta_{j}^{0}\right|\right)\left(1+\sum_{j}\left(\operatorname{Re} \zeta_{j}^{0}\right)^{-1}\right)\right]^{k t^{\prime}}
$$


for some sequence $c_{k}$, such that $c_{k} \leqq \alpha \beta^{k^{2}}$ for some $\alpha, \beta>0$, some positive integer $t^{\prime}$, not depending on $k$ and $r$; and all $k=1,2, \ldots$.

The subsets $C_{k}^{(r)}$ are increasing: $C_{k}^{(r)} C C_{k}^{(r+1)} ; C_{k}^{(0)}$ is just the $k$ fold product of the positive real axis and most importantly, the union $\bigcup_{r} C_{k}^{(r)}$ of all the subsets is all of $\mathbb{C}_{+}^{k}$. Parenthetically we remark that only the bounds $T E_{r}$ require the linear growth condition (4.2). For the other results the original temperedness condition (EO) of OS I is sufficient.

The final result of our induction is summarized in the next theorem.

Theorem 4.3. There are functions $S_{k}\left(\underline{\zeta}^{0} \mid \underline{\xi}\right)$, analytic in the variables $\underline{\zeta}^{0}$, continuous in the variables $\underline{\xi}$ for $\underline{\zeta}^{0} \in \mathbb{C}_{+}^{k}$ and $\underline{\vec{\xi}} \in \mathbb{R}^{3 k}$, such that (4.4) and (4.6) hold.

By standard arguments (see Vladimirov [19], p. $235 \mathrm{ff}$.) Theorem 4.3 implies that there exist unique distributions $\hat{W}_{k} \in \mathscr{S}^{\prime}\left(\mathbb{R}^{4 k}\right)$ with support in $\overline{\mathbb{R}_{+}^{4 k}}$ such that $S_{k}$ are the Fourier-Laplace transform of them:

$$
S_{k}\left(\underline{\zeta}^{0} \mid \underline{\vec{\xi}}\right)=\int \hat{W}_{k}(\underline{q}) \exp \left[-\sum_{j=1}^{k}\left(\zeta_{j}^{0} q_{j}^{0}-i \vec{\xi}_{j} \vec{q}_{j}\right)\right] d^{4 k} q .
$$

As in OS I we conclude that $\tilde{W}_{k}$ is the Fourier transform of the difference variable Wightman distribution $W_{k}$.

Furthermore, again using (4.6), we find that for $h \in \mathscr{S}\left(\mathbb{R}^{4 k}\right)$,

$$
\begin{aligned}
W_{k}(h) & \equiv \int W_{k}(\underline{\xi}) h(\underline{\xi}) d^{4 k} \xi \\
& =\lim _{\eta_{i}^{0} \rightarrow 0+} \int S_{k}\left(\eta^{0}+i \underline{\xi^{0}} \mid \underline{\vec{\xi}}\right) h(\underline{\xi}) d^{4 k} \xi
\end{aligned}
$$

satisfies the inequality

$$
\left|W_{k}(h)\right| \leqq w_{k}^{\prime}|h|_{k t^{\prime \prime}}
$$

for some sequence $w_{k}^{\prime} \leqq \alpha^{\prime}\left(\beta^{\prime}\right)^{k^{2}}$ and $t^{\prime \prime}=\frac{3 t}{2}+5$, see Vladimirov [19], p. 235, Eq. (14).

It remains to derive $R 0^{\prime}$ from (4.7). Let $f \in \mathscr{S}\left(\mathbb{R}^{4 n}\right)$ and set $h_{x_{1}}(\underline{\xi})=f\left(x_{1}, x_{2} \ldots x_{n}\right)$ where $\xi_{k}=x_{k+1}-x_{k}$ for $k=1, \ldots n-1$. Then

$$
\begin{aligned}
\left|\mathfrak{W}_{n}(f)\right| & =\left|\int W_{n-1}\left(h_{x_{1}}\right) d^{4} x_{1}\right| \\
& \leqq w_{n-1}^{\prime} \int\left|h_{x_{1}}\right|_{k t^{\prime \prime}} d^{4} x_{1} \leqq \omega_{n}|f|_{k t^{\prime \prime \prime}}
\end{aligned}
$$

for some new sequence $\omega_{n} \leqq \alpha^{\prime \prime}\left(\beta^{\prime \prime}\right)^{n^{2}}$ and $t^{\prime \prime \prime}=t^{\prime \prime}+4$. This concludes our proof of Theorem $E^{\prime} \rightarrow R^{\prime}$.

In the remaining chapters we explain the inductive procedures in detail and prove Theorems 4.1 and 4.2 .

\section{The Analytic Continuation}

In this chapter we construct the analytic continuation of the Euclidean Green's functions to "real times" and prove $\left(A_{r}\right)$ for $r=0,1,2, \ldots$. No use will be made of the linear growth condition $E 0^{\prime}$ at this point; all we assume for the moment is $E 0, E 1$ (invariance) and $E 2$ (positivity). 
As we have seen in Section IV.2 we have to analytically continue $S_{k}(\underline{\xi}) \equiv S_{k}\left(\underline{\xi^{0}} \mid \underline{\vec{\xi}}\right)$ in the "time variables" $\xi^{0}$ only; the "spatial variables" $\underline{\xi}$ play the role of parameters. There are two different ways of dealing with the spatial variables in a rigorous fashion:

Method $A$ : To treat the spatial variables as distributional variables throughout. More precisely for $f_{i k} \in \mathscr{S}\left(\mathbb{R}^{3}\right)$ and $\underline{f}^{n}=\left(f_{1 n}, \ldots, f_{n n}\right)$ we define with $\xi_{i}^{0}=x_{i+1}^{0}-x_{i}^{0}$,

$$
S_{n-1}\left(\underline{\xi}^{0} \mid \underline{f}^{n}\right)=\int \mathfrak{S}_{n}(\underline{x}) f_{1 n}\left(\vec{x}_{1}\right) \ldots f_{n n}\left(\vec{x}_{n}\right) d^{3 n} x .
$$

Positivity will play an important role in this and the next chapter, and $S_{n-1}\left(\xi^{0} \mid \underline{f}\right)$ was defined such that it still satisfies a positivity condition:

$$
\sum_{n, m} \int \bar{h}_{n}\left(x^{0}, \underline{\xi}^{0}\right) S_{n+m-1}\left(\underline{\xi^{0}}, x^{0}+x^{0^{\prime}}, \underline{\xi}^{0^{\prime}} \mid \vartheta \underline{f}^{n} \times \underline{f}^{m}\right) \cdot h_{m}\left(x^{0^{\prime}}, \underline{\xi}^{0^{\prime}}\right) d x^{0} d x^{0^{\prime}} d \underline{\xi}^{0} d \underline{\xi}^{0^{\prime}} \geqq 0
$$

for all finite sequences $\left\{h_{0}, h_{1}, \ldots\right\}, h_{0} \in \mathbb{C}, h_{n} \in \mathscr{S}\left(\mathbb{R}_{+}^{n}\right)$ and all $f_{i k} \in \mathscr{S}\left(\mathbb{R}^{3}\right)$, where $\vartheta f^{n} \times f^{m}$ is the function

$$
\bar{f}_{n n}\left(\vec{x}_{1}\right) \ldots \bar{f}_{1 n}\left(\vec{x}_{n}\right) f_{1 m}\left(\vec{x}_{n+1}\right) \ldots f_{m m}\left(\vec{x}_{n+m}\right)
$$

and $\underline{\xi}^{0}=\left(\xi_{n-1}^{0}, \xi_{n-2}^{0}, \ldots \xi_{1}^{0}\right)$

$\bar{M}$ ethod B: To show that $S_{k}\left(\underline{\xi}^{0} \mid \underline{\xi}\right)$ can be regarded as a continuous function of the spatial variables; this makes smearing out redundant.

Method A was sketched in [13]. It is simple, mainly because it allows for the reconstruction of the Wightman distributions without using $\mathrm{SO}_{4}$ invariance of the Euclidean Green's functions. The drawback of this method is that in order to derive a temperedness estimate for the analytically continued Euclidean Green's functions, we need a distribution assumption slightly stronger than $E 0^{\prime}$, such as $E 0^{\prime \prime}$. Though it is true that $E 0^{\prime \prime}$ is most probably satisfied in all reasonable quantum field theory models - see Glimm and Jaffe [7] - the weaker assumption $E 0^{\prime}$ is more satisfactory from an axiomatic point of view: besides being more general it does not make use of coinciding arguments of the Euclidean Green's functions. This justifies our use of the more complicated method B in this paper. Extending a geometrical argument of Glaser [6] we use $\mathrm{SO}_{4}$ invariance to prove that the $S_{k}$ are real analytic functions in all variables. Then we derive a temperedness estimate for these functions from their behaviour as distributions.

Let us first summarize some results of OS I (in a slightly changed notation). In terms of the difference variable Euclidean Green's functions $S_{k}$ the positivity axiom $E 2$ requires that for all finite sequences $\left\{f_{0}, f_{1}, f_{2}, \ldots\right\}, f_{0} \in \mathbb{C}, f_{n} \in \mathscr{S}\left(\mathbb{R}_{+}^{4 k}\right)$

$$
\begin{aligned}
\sum_{n, m} \int \bar{f}_{n}(x, \underline{\xi}) S_{n+m-1}\left(-\overleftarrow{\vartheta} \underline{\xi},-\vartheta x+x^{\prime}, \underline{\xi}^{\prime}\right) f_{m}\left(x^{\prime}, \underline{\xi}^{\prime}\right) d^{4} x d^{4} x^{\prime} d^{4(n-1)} \xi d^{4(m-1)} \xi^{\prime} \\
\quad \equiv \sum_{n, m} S_{n+m-1}\left(f_{n}, f_{m}\right) \geqq 0
\end{aligned}
$$

where $\underline{\xi}=\left(\xi_{1}, \ldots \xi_{n-1}\right), \xi^{\prime}=\left(\xi_{1}^{\prime}, \ldots \xi_{m-1}^{\prime}\right), \vartheta \underline{\xi}=\left(\vartheta \xi_{1}, \ldots \vartheta \xi_{n-1}\right), \vartheta \xi_{k}=\left(-\xi_{k}^{0}, \vec{\xi}_{k}\right)$ and finally $\underline{\bar{\xi}}=\left(\xi_{n-1}, \ldots \xi_{1}\right)$.

As in OS I we construct a Hilbert space $\mathscr{H}$ and furthermore $\mathscr{H}$-valued distributions $\Psi_{n}(x, \underline{\xi})$ such that for $f \in \mathscr{S}\left(\mathbb{R}_{+}^{4 n}\right), g \in \mathscr{S}\left(\mathbb{R}_{+}^{4 m}\right)$

$$
\Psi_{n}(f)=\int \Psi_{n}(x, \underline{\xi}) f(x, \underline{\xi}) d^{4} x d^{4(n-1)} \xi \in \mathscr{H}
$$

with scalar product

$$
\left(\Psi_{n}(f), \Psi_{m}(g)\right)=S_{n+m-1}(f, g),
$$


or, in the sense of distributions,

$$
\left(\Psi_{n}(x, \underline{\xi}), \Psi_{m}\left(x^{\prime}, \underline{\xi}^{\prime}\right)\right)=S_{n+m-1}\left(-\overleftarrow{\vartheta} \underline{\xi},-\vartheta x+x^{\prime}, \underline{\xi}^{\prime}\right)
$$

The set of vectors $\Psi_{n}(f), f \in \mathscr{S}\left(\mathbb{R}_{+}^{4 n}\right), n=0,1,2, \ldots$ (for $n=0, f$ must be in $\mathbb{C}$, of course) is total in $\mathscr{H}$.

By the arguments of OS I, we can construct a weakly continuous semigroup of self-adjoint contractions $e^{-t H}$ on $\mathscr{H}, t \geqq 0, H=H^{*} \geqq 0$, such that (in the sense of distributions)

$$
e^{-t H} \Psi_{n}(x, \underline{\xi})=\Psi_{n}\left(\left(x^{0}+t, \vec{x}\right), \underline{\xi}\right) .
$$

Furthermore for $\tau \in \mathbb{C}_{+}=(0, \infty)+i \mathbb{R}$

$$
\left(\Psi_{n}(x, \underline{\xi}), e^{-\tau H} \Psi_{m}\left(x^{\prime}, \underline{\xi^{\prime}}\right)\right) \equiv S_{n+m-1}\left(-\overleftarrow{\vartheta^{\xi}} \underline{\underline{\xi}},\left(x^{0}+x^{0 \prime}+\tau,-\vec{x}+\vec{x}^{\prime}\right), \underline{\xi}^{\prime}\right)
$$

defines an analytic continuation of $S_{n+m-1}$ in the $n$ 'th time variable: by OS I the right hand side of (5.4) is an analytic function of the variable $z=x^{0}+x^{0^{\prime}}+\tau$ for $\mathscr{R} e z>0$, while still being a distribution in all the remaining variables. (It was at this point in OS I that the wrong Lemma 8.8 was used to continue $S_{k}$ in all the time variables simultaneously to the $k$-fold product of $\mathbb{C}_{+}$.) In the following we use (5.4) and Euclidean covariance $E 2$ to show that $S_{k}(\xi)$ is the restriction of a function $S_{k}(\zeta)$, analytic in a complex neighborhood of $\mathbb{R}_{+}^{4 \bar{k}}$, i.e. assertion $\left(A_{0}\right)$.

\section{V.I. Real Analyticity}

For $0<\gamma<\pi / 4$ we define

and

$$
\mathbb{R}_{+}^{4}(\gamma)=\left\{x=\left(x^{0}, \vec{x}\right)\left|x^{0}>\right| \vec{x} \mid \operatorname{tg} \gamma\right\},
$$

$$
\mathbb{R}_{+}^{4 n}(\gamma)=\left\{\underline{x}=\left(x_{1}, \ldots x_{n}\right) \mid x_{k} \in \mathbb{R}_{+}^{4}(\gamma), k=1, \ldots n\right\} .
$$

Obviously $\mathbb{R}_{+}^{4}(\gamma)$ is the largest cone in $\mathbb{R}_{+}^{4}$ which under an arbitrary rotation $\mathscr{R}(a, \varphi)$ about an axis $a$ through the origin, by an angle $\varphi \leqq \gamma$, stays in $\mathbb{R}_{+}^{4}$. Euclidean covariance implies that for any $\underline{\xi} \in \mathbb{R}_{+}^{4 k}(\gamma)$ and $0 \leqq \varphi \leqq \gamma$

$$
S_{k}(\mathscr{R}(a, \varphi) \underline{\xi})=S_{k}(\underline{\xi})
$$

where $\mathscr{R}(a, \varphi) \underline{\xi}=\left(\mathscr{R}(a, \varphi) \xi_{1}, \ldots \mathscr{R}(a, \varphi) \xi_{k}\right)$.

For fixed $\gamma \in(0, \pi / 4)$ let $e_{\mu}=e_{\mu}(\gamma), \mu=1, \ldots 4$, be four linearly independent vectors in $\mathbb{R}_{+}^{4}(\pi / 2-\gamma)$, the dual cone of $\mathbb{R}_{+}^{4}(\gamma)$. Then there are rotations $\mathscr{R}_{\mu}=\mathscr{R}\left(a_{\mu}, \varphi_{\mu}\right)$ with $0 \leqq \varphi_{\mu}<\gamma$ such that

$$
\mathscr{R}_{\mu} e_{\mu}=(1,0,0,0) \text {. }
$$

Now let $\underline{\xi} \in \mathbb{R}_{+}^{4 k}(\gamma)$ be fixed and $\underline{u}=\left(u_{1}, \ldots u_{k}\right)$, where $u_{i}=\left(u_{i}^{1}, u_{i}^{2}, u_{i}^{3}, u_{i}^{4}\right)$, $u_{i}^{\mu} \in[0, \infty)$. Writing $\underline{u} \cdot e$ for the vector $\left(\sum_{1}^{4} u_{1}^{\mu} e_{\mu}, \ldots \sum_{1}^{4} u_{k}^{\mu} e_{\mu}\right)$ in $\mathbb{R}_{+}^{4 k}(\pi / 2-\gamma)$ we
now consider

$$
S_{k}(\underline{0} \underline{\xi}+\underline{u} \cdot e)
$$

as a distribution in the variables $u_{n}^{\mu}$. By (5.5) we find that for $\mu=1, \ldots 4$

$$
S_{k}(\underline{\underline{\xi}}+\underline{u} \cdot e)=S_{k}\left(\mathscr{R}_{\mu} \underline{\underline{\xi}}+\underline{u} \cdot \mathscr{R}_{\mu} e\right)
$$

where $\mathscr{R}_{\mu} e$ stands for the four vectors $\mathscr{R}_{\mu} e_{v}, v=1, \ldots 4$. By (5.4) and (5.6) the right hand side of (5.7) can be analytically continued to $\mathbb{C}_{+}$in each of the variables $u_{i}^{\mu}, i=1, \ldots k$, (one at a time), while it is still a distribution in all the other $u$-variables. 
Now we pass to variables $s_{i}^{\mu}=\ln u_{i}^{\mu}$ and set $\hat{T}\left(s_{1}^{1}, \ldots s_{k}^{4}\right)=S_{k}(\underline{0} \underline{\xi}+\underline{u} \cdot e)$. According to the above argument we can find functions

$$
T_{i \mu}\left(s_{1}^{1}, \ldots, r_{i}^{\mu}, \ldots s_{k}^{4}\right)
$$

which are analytic in $r_{i}^{\mu}=s_{i}^{\mu}+i t_{i}^{\mu},\left|t_{i}^{\mu}\right|<\pi / 2$, and have values in $\mathscr{D}^{\prime}$ with respect to the variables $s_{1}^{1}, \ldots s_{i}^{\mu}, \ldots s_{k}^{4}$. All the $T_{i \mu}$ analytically continue the same distribution $\hat{T}$. It follows now from the Malgrange-Zerner theorem, see Epstein [2], which deals with a degenerate case of the well known "tube theorem" (see e.g. Vladimirov [19], p. 154), that there is an analytic function

$$
T(\underline{r})=T\left(r_{1}^{1}, \ldots, r_{k}^{4}\right)
$$

analytic in the convex envelope $\mathscr{T}$ of the union of all the flat tubes

$$
\mathscr{T}_{i \mu}=\left\{r_{1}^{1} \ldots r_{k}^{4}\left|\operatorname{Im} r_{i}^{\mu}\right|<\pi / 2, r_{j}^{\nu}=0 \quad \text { for } \quad v \neq \mu \quad \text { or } j \neq i\right\},
$$

such that $T$ continues all the $T_{i \mu}$. We find that $\mathscr{T}=\left\{r_{1}^{1}, \ldots r_{k}^{4}\left|\sum_{i, \mu}\right| \operatorname{Im} r_{i}^{\mu} \mid<\pi / 2\right\}$. Going back to the variables $u_{i}^{\mu}$ and to $S$ we have therefore shown that there exists a function

analytic in

$$
\begin{gathered}
S_{k}(\underline{0} \underline{\xi}+\underline{w} \cdot e), \\
\left\{\underline{w}\left|\sum_{i, \mu}\right| \arg w_{i}^{\mu} \mid<\pi / 2\right\},
\end{gathered}
$$

whose restriction to real arguments defines the distribution $S_{k}(\underline{0} \dot{\xi}+\underline{u} \cdot e)$, with $\underline{0}$ and $e_{1}, \ldots e_{4}$ playing the role of fixed parameter. Assertion $\left(A_{0}\right)$ is now an immediate consequence.

For the benefit of Section VI.1, where we establish the bound $\left(T E_{0}\right)$, we now derive an estimate on the size of the region of analyticity of the function $S_{k}(\underline{\zeta})$ obtained above.

Lemma 5.1. For fixed $\underline{\xi} \in \mathbb{R}_{+}^{4 k}$, the functions $S_{k}(\underline{\xi}+\underline{\zeta})$ are analytic in the polydisc

where

$$
\Gamma(\underline{\xi})=\left\{\underline{\zeta}|| \zeta_{i}^{\mu} \mid \leqq \varrho, \text { for } 1 \leqq i \leqq k, 1 \leqq \mu \leqq 4\right\} \text {, }
$$

$$
\varrho==\frac{c}{k}\left(1+\max _{1 \leqq j \leqq k}\left|\vec{\xi}_{j}\right|\right)^{-1}\left(1+\sum_{j}\left(\xi_{j}^{0}\right)^{-1}\right)^{-2}
$$

for some constant $c \in(0,1)$ independent of $k$ or $\underline{\xi}$.

Proof. For given $\underline{\xi}$ we define $\gamma$ and $\stackrel{0}{\xi}$ by

$$
\begin{aligned}
& \operatorname{tg} 2 \gamma=\min _{1 \leqq j \leqq k} \xi_{j}^{0} /\left|\vec{\xi}_{j}\right| \quad \text { and } \\
& \underline{0}=\left(\stackrel{0}{\xi}_{1}^{0}, \ldots \stackrel{0}{\xi}_{k}\right), \quad \stackrel{0}{\xi}_{i}=\left(\frac{1}{2} \xi_{i}^{0}, \vec{\xi}_{i}\right), \quad 1 \leqq i \leqq k,
\end{aligned}
$$

and we choose the vectors $e_{1}, \ldots e_{4}$ as follows:

$$
\begin{aligned}
& e_{1}=(2 \operatorname{ctg} \gamma, 1,1,1) \\
& e_{2}=(2 \operatorname{ctg} \gamma, 1,-1,-1) \\
& e_{3}=(2 \operatorname{ctg} \gamma,-1,1,-1) \\
& e_{4}=(2 \operatorname{ctg} \gamma,-1,-1,1) .
\end{aligned}
$$


Obviously $0<\gamma<\pi / 4, \underline{\xi} \in \mathbb{R}_{+}^{4 k}(\gamma), \quad e_{\mu} \in \mathbb{R}_{+}^{4 k}(\pi / 2-\gamma)$ and $e_{1}, \ldots e_{4}$ are linearly independent. (5.11) implies

and

$$
\begin{aligned}
& (1,0,0,0)=2^{-3} \operatorname{tg} \gamma\left(e_{1}+e_{2}+e_{3}+e_{4}\right) \\
& (0,1,0,0)=2^{-2}\left(e_{1}+e_{2}-e_{3}-e_{4}\right) \\
& (0,0,1,0)=2^{-2}\left(e_{1}-e_{2}+e_{3}-e_{4}\right) \\
& (0,0,0,1)=2^{-2}\left(e_{1}-e_{2}-e_{3}+e_{4}\right)
\end{aligned}
$$

where $\sigma_{r \mu}$ is equal to +1 or -1 and may be read off Eq. (5.11'). It follows from (5.8) that $S_{k}(\underline{\xi}+\underline{\zeta})$ is analytic for those values of $\underline{\zeta}$ for which $\sum_{i, \mu}\left|\arg w_{i}^{\mu}\right|<\pi / 2$. It therefore suffices to assume that for all $i, \mu\left|\arg w_{i}^{\mu}\right|<\frac{\pi}{2^{3} k}$, which is implied by

$$
\frac{\left|\operatorname{Im} w_{i}^{\mu}\right|}{\left|\operatorname{Re} w_{i}^{\mu}\right|}<\frac{\pi}{2^{3} k} .
$$

By (5.12) the $w_{i}^{\mu}$ are given as functions of the $\zeta_{i}^{\mu}$, and (5.13) is satisfied if we restrict $\zeta_{i}^{\mu}$ by

$$
\left|\zeta_{i}^{\mu}\right|<\frac{\pi}{2^{3} k} \cdot 2^{-5} \xi_{i}^{0} \operatorname{tg} \gamma
$$

By (5.10), $\operatorname{tg} \gamma<\frac{1}{2} \operatorname{tg} 2 \gamma=\frac{1}{2} \min _{1 \leqq j \leqq k}\left(\xi_{j}^{0} /\left|\vec{\xi}_{j}\right|\right)$ and hence $S_{k}(\underline{\xi}+\underline{\zeta})$ is analytic if

$$
\left|\zeta_{i}^{\mu}\right|<\frac{\pi}{2^{9} \cdot k} \xi_{i}^{0} \cdot \min _{j}\left(\xi_{j}^{0} /\left|\vec{\xi}_{j}\right|\right)
$$

This implies Lemma 5.1, with $c=2^{-9} \pi$.

\section{V.2. Towards the Real World}

Having established the real analyticity of $S_{k}(\underline{\xi})$ we now proceed to construct the analytic continuation $S_{k}\left(\underline{\zeta}^{0} \mid \underline{\xi}\right)$ of $S_{k}$ in the time variables to the $n$-fold product of $\mathbb{C}_{+}$. (Notice that $i \zeta_{n}^{0}$ are actually the times, hence at the boundary of $\mathbb{C}_{+}$we will arrive at real times.) Our method is to verify inductively the following sequences of statements:

$\left(A_{N}\right)$ There are analytic continuations $S_{k}\left(\underline{\zeta}^{0} \mid \underline{\vec{\xi}}\right)$ of $S_{k}\left(\underline{\xi}^{0} \mid \underline{\vec{\xi}}\right)$, which are continuous in $\underline{\xi} \in \mathbb{R}^{3 k}$ and analytic in $\underline{\zeta}^{0} \in C_{k}^{(N)} C \mathbb{C}_{+}^{k}$. For $N=1,2, \ldots, C_{k}^{(N)}$ is the envelope of holomorphy of

$$
\hat{C}_{k}^{(N)} \equiv \bigcup_{n=1}^{k}\left\{\left(\underline{\overleftarrow{\zeta}}, x^{0}+x^{0 \prime}+z, \underline{\zeta}^{0 \prime}\right) \mid\left(x^{0}, \underline{\zeta}^{0}\right) \in D_{n}^{(N-1)},\left(x^{0^{\prime}}, \underline{\zeta}^{0 \prime}\right) \in D_{k-n+1}^{(N-1)}, z \in \mathbb{C}_{+}\right\}
$$


$\left(P_{N}\right) \quad$ There are $\mathscr{H}$-valued functions

$$
\Psi_{n}\left(x^{0}, \underline{\zeta}^{0} \mid \vec{x}, \underline{\vec{\xi}}\right)
$$

defined for $(\vec{x}, \underline{\xi}) \in \mathbb{R}^{3 n}$ and $\left(x^{0}, \underline{\zeta}^{0}\right) \in D_{n}^{(N)} \subset(0, \infty) \times \mathbb{C}_{+}^{n-1}$, where for $N=1,2, \ldots$

$$
D_{n}^{(N)}=\left\{\left(x^{0}, \underline{\zeta}^{0}\right) \mid x^{0}>0,\left(\underline{\zeta}^{0}, 2 x^{0}, \underline{\zeta}^{0}\right) \in C_{2 n-1}^{(N)}\right\},
$$

such that the scalar product is given by

$$
\left(\Psi_{n}\left(x^{0}, \underline{\zeta}^{0} \mid \vec{x}, \vec{\xi}\right), \Psi_{m}\left(x^{0^{\prime}}, \underline{\zeta}^{0 \prime} \mid \vec{x}^{\prime}, \vec{\xi}^{\prime}\right)\right)=S_{n+m-1}\left(\underline{\overleftarrow{\zeta^{0}}}, x^{0}+x^{0 \prime}, \underline{\zeta}^{0 \prime} \mid-\underline{\overleftarrow{\xi}},-\vec{x}+\vec{x}^{\prime}, \underline{\vec{\xi}}\right) .
$$

Notice that the passage from $N-1$ to $N$ takes place in (5.15), where $C_{k}^{(N)}$ is defined in terms of the regions $D_{n}^{(N-1)}$. Later we will show that $\bigcup_{N} C_{k}^{(N)}=\mathbb{C}_{+}^{k}$, which
completes the analytic continuation.

In the rest of this chapter the spatial variables will always play the role of parameters and we therefore drop them completely in our formulas. Continuity with respect to them will be evident at each step. Also we will drop the superscripts ${ }^{0}$, hence $\zeta$ will now stand for $\left(\zeta_{1}^{0}, \ldots \zeta_{k}^{0}\right)$, etc.

To start the induction we set

$$
\begin{aligned}
& C_{k}^{(0)}=\left\{\underline{\zeta} \mid \arg \zeta_{i}=0, i=1, \ldots k\right\}, \\
& D_{n}^{(0)}=\left\{(x, \underline{\zeta}) \mid x>0, \arg \zeta_{i}=0, i=1, \ldots n-1\right\} .
\end{aligned}
$$

Then $\left(A_{0}\right)$ follows from the results of Section V.1. We claim that $\left(P_{0}\right)$ follows from $\left(A_{0}\right)$ and (5.2). Notice that (5.2) was valid in the sense of distributions only, while $\left(P_{0}\right)$ asserts that it also holds in the sense of functions, i.e., pointwise. For a proof smear both sides of (5.2) with two functions $f_{v} \in \mathscr{S}\left(\mathbb{R}_{+}^{4 n}\right), g_{v} \in \mathscr{S}\left(\mathbb{R}_{+}^{4 n}\right)$, which as $v$ tends to infinity tend to $\delta$-functions and take the limit.

Now assume $\left(A_{N}\right)$ and $\left(P_{N}\right)$ have been verified for $0 \leqq N \leqq M-1$. We will prove $\left(A_{M}\right)$ and $\left(P_{M}\right)$.

By $\left(P_{M-1}\right)$ we can define

$$
S_{n+m-1}\left(\underline{\bar{\zeta}}, x+x^{\prime}+z, \underline{\zeta}^{\prime}\right)=\left(\Psi_{n}(x, \underline{\zeta}), e^{-z H} \Psi_{m}\left(x^{\prime}, \underline{\zeta}^{\prime}\right)\right)
$$

for $(x, \zeta) \in D_{n}^{(M-1)}$ and $\left(x^{\prime}, \underline{\zeta}^{\prime}\right) \in D_{m}^{(M-1)}$; this analytically extends $S_{k}, k=n+m-1$, to $\bar{C}_{k}^{(M)}$ and hence, because $C_{k}^{(M)}$ is the envelope of holomorphy of $C_{k}^{(M)}$, there is an analytic extension of $S_{k}$ into $C_{k}^{(M)}$. This proves $\left(A_{M}\right)$.

Now take a point $(\stackrel{0}{x}, \stackrel{0}{\zeta}) \in D_{n}^{(M)}$, defined by $(5.16)$, and observe that with $(\stackrel{0}{x}, \stackrel{0}{\zeta})$ the whole cone of points of the form $(x, \zeta)$ with $x>0,\left|\arg \zeta_{i}\right| \leqq\left|\arg \stackrel{0}{\zeta}_{i}^{0}\right|, i=1, \ldots n-1$, is contained in $D_{n}^{(M)}$. We therefore can find points $\xi_{i} \in(0, \infty)$ and numbers $r_{i}>0$, such that the whole polydisc

$$
P=\left\{(x, \underline{\zeta})|x=\stackrel{0}{x},| \zeta_{i}-\xi_{i} \mid<r_{i}, i=1, \ldots n-1\right\}
$$

is contained in $D_{n}^{(M)}$ and $(\stackrel{0}{x}, \stackrel{0}{\zeta}) \in P$, see figure below.

Now we define the vector $\Psi_{n}(\stackrel{0}{x}, \stackrel{0}{\zeta})$ by

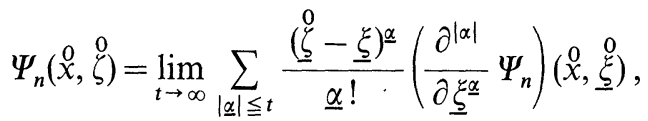




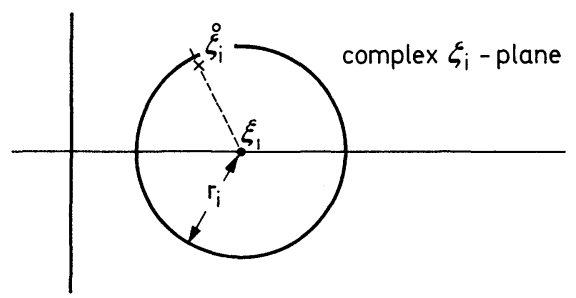

Fig. 1

where the derivatives of the vector $\Psi_{n}$ are taken in the strong Hilbert space topology. The right hand side of (5.20) converges in norm, for

$$
\begin{aligned}
& \left\|\sum_{t<|\alpha|} \frac{(\underline{\underline{\xi}}-\underline{\xi})^{\underline{\alpha}}}{\underline{\alpha} !}\left(\frac{\partial^{|\alpha|}}{\partial \xi^{\underline{\alpha}}} \Psi_{n}\right)\left(x^{0}, \underline{\xi}\right)\right\|^{2} \\
& =\left.\sum_{\substack{t<|\alpha| \\
t<|\beta|}} \frac{(\underline{\zeta}-\underline{\xi})^{\underline{\alpha}}}{\underline{\alpha} !} \frac{(\underline{\zeta}-\underline{\xi})^{\underline{\beta}}}{\underline{\beta} !} \frac{\partial^{|\alpha|}}{\partial \underline{\xi}^{\underline{\alpha}}} \frac{\partial^{|\beta|}}{\partial \underline{\xi^{\prime}} \underline{\underline{\beta}}} S_{2 n-1}\left(\underline{\xi}, 2 \underline{\tilde{x}}^{0}, \underline{\xi^{\prime}}\right)\right|_{\underline{\xi}=\underline{\xi^{\prime}}}
\end{aligned}
$$

and the right hand side of (5.21) is just the remainder term of the Taylor expansion of $S_{2 n-1}(\underline{\xi}, 2 \stackrel{0}{x}, \underline{0})$ about the point $(\underline{\xi}, 2 \dot{x}, \underline{\xi})$ and thus tends to zero as we let $t$ go to infinite. Relation (5.17) follows easily from (5.20). This establishes $\left(P_{M}\right)$.

Finally we have to prove that $\bigcup_{N} C_{k}^{(N)}=\mathbb{C}_{+}^{k}$. But this is a consequence of the following stronger result, which we will need in Section VI.2.

Lemma 5.2. For all $N, n, k \in \mathbb{Z}_{+}$,

(a) $D_{n}^{(N)}$ contains the set

$$
\left\{(x, \underline{\zeta})|x>0,| \arg \zeta_{i} \mid<\varrho(i, N), 1 \leqq i \leqq n-1\right\},
$$

(b) $C_{k}^{(N)}$ contains the set

where

$$
\left\{\zeta|| \arg \zeta_{i} \mid<\varrho(i, N), 1 \leqq i \leqq k\right\},
$$

and

$$
\varrho(i, N)=\frac{\pi}{2}\left[1-2^{-N} \sum_{t=0}^{i-1}\left(\begin{array}{c}
N \\
t
\end{array}\right)\right]
$$

$$
\sum_{t=0}^{i-1}\left(\begin{array}{c}
N \\
t
\end{array}\right) \equiv 2^{N} \quad \text { for } \quad i>N
$$

Proof. By construction the regions $C_{k}^{(N)}$ and $D_{n}^{(N)}$ are mapped onto tubular domains under the transformation $\zeta_{i}=e^{w_{i}}=e^{u_{i}+i v_{i}}$. We define $c_{k}^{(N)}$ and $d_{n}^{(N)}$ to be the closure of the bases of these tubes:

$$
\begin{aligned}
& c_{k}^{(N)}=\left\{\underline{v} \mid v_{i}=\operatorname{Im} w_{i}, 1 \leqq i \leqq k,\left(e^{w_{1}} \ldots e^{w_{k}}\right) \in C_{k}^{(N)}\right\}^{-} \\
& d_{n}^{(N)}=\left\{(0, \underline{v}) \mid v_{i}=\operatorname{Im} w_{i}, 1 \leqq i \leqq n-1,\left(e^{u_{0}}, e^{w_{1}}, \ldots e^{w_{n-1}}\right) \in D_{n}^{(N)}\right\}^{-} .
\end{aligned}
$$

Note that $c_{k}^{(N)}$ and $d_{n}^{(N)}$ are subsets of $[-\pi / 2, \pi / 2]^{k}$ and of $[-\pi / 2, \pi / 2]^{n}$ respectively. 
From the inductive definitions $(5.15 / 16),(5.18 / 19)$, and from the tube theorem we find for $r=1,2 \ldots$

$c_{k}^{(N)}=$ convex hull of

$$
\left\{\underline{v} \mid \underline{v}=\left(-\underline{\underline{t}}^{\prime}, v, v^{\prime \prime}\right), \text { where }\left(0, \underline{v}^{\prime}\right) \in d_{n}^{(N-1)},\left(0, \underline{v}^{\prime \prime}\right) \in d_{k-n+1}^{(N-1)},|v| \leqq \pi / 2\right\}
$$

$d_{n}^{(N)}=\left\{(0, \underline{v}) \mid(-\underline{\underline{t}}, 0, \underline{v}) \in c_{2 n-1}^{(N)}\right\}$

and

$$
c_{k}^{(0)}=\{(0, \ldots 0)\}, d_{n}^{(0)}=\{(0, \ldots 0)\} .
$$

Observe that all the sets $c_{k}^{(N)}$ and $d_{k}^{(N)}$ are convex. Moreover if $\left(v_{1}, \ldots v_{k}\right) \in c_{k}^{(N)}$ (or $d_{k}^{(N)}$ ), then the whole hyperrectangle with corners $\left( \pm v_{1}, \ldots \pm v_{k}\right)$ is also contained in $c_{k}^{(N)}\left(d_{k}^{(N)}\right.$ resp.).

For a proof of Lemma 5.2 we show inductively that the points $\left(0, v_{1}, \ldots v_{n-1}\right)$ with $\left|v_{i}\right|=\varrho(i, N)$ are in $d_{n}^{(N)}$. This establishes part (a); part (b) follows from (a) and the equation $S_{k}(\underline{\zeta})=\left(\Omega, \Psi_{k+1}(x, \underline{\zeta})\right)$.

We first construct a function $\bar{h}_{i}^{N}, i=1,2, \ldots, N=1,2, \ldots$, such that for all $s \geqq 0, t \geqq 1$,

$$
w^{N}(s, t) \equiv(\underbrace{0,0, \ldots 0}_{t}, h_{1}^{N}, h_{2}^{N}, \ldots h_{s}^{N}) \in d_{t+s}^{(N)} .
$$

We choose $h_{i}^{0}=0$ for all $i$. By $(5.25), w^{0}(s, t) \in d_{t+s}^{(0)}$. Suppose now we have already constructed $h_{i}^{N}$ for all $i \geqq 1$ and $N=1, \ldots M$, such that (5.26) holds. Then by (5.23) the following points are contained in $c_{2(s+t)-1}^{(M+1)}$

and

$$
(-h_{s}^{M}, \ldots-h_{2}^{M},-h_{1}^{M}, \underbrace{0, \ldots 0}_{2 t-1}, \pi / 2, h_{1}^{M}, \ldots h_{s-1}^{M})
$$

$$
(-h_{s-1}^{M} \cdots-h_{1}^{M},-\pi / 2, \underbrace{0, \ldots 0}_{2 t-1}, h_{1}^{M}, h_{2}^{M}, \ldots h_{s}^{M}) .
$$

Because $c_{2(s+t)-1}^{(M+1)}$ is convex it also contains the point

$$
\begin{aligned}
&\left(-\frac{1}{2}\left(h_{s}^{M}+h_{s-1}^{M}\right), \cdots-\frac{1}{2}\left(h_{2}^{M}+h_{1}^{M}\right),-\frac{1}{2}\left(h_{1}^{M}+\pi / 2\right),\right. \underset{2 t-1}{0, \ldots 0}, \frac{1}{2}\left(\pi / 2+h_{1}^{M}\right), \\
&\left.\frac{1}{2}\left(h_{1}^{M}+h_{2}^{M}\right), \ldots \frac{1}{2}\left(h_{s-1}^{M}+h_{s}^{M}\right)\right) .
\end{aligned}
$$

This means [by (5.24)] that with

$$
\begin{aligned}
h_{i}^{0} & =0, & & i=1,2, \ldots \\
h_{1}^{M+1} & =\frac{1}{2}\left(h_{1}^{M}+\pi / 2\right) & & \\
h_{i}^{M+1} & =\frac{1}{2}\left(h_{i}^{M}+h_{i-1}^{M}\right), & & i=2,3, \ldots
\end{aligned}
$$

the points $w^{M+1}(s, t)$ defined by (5.26) are again in $d_{t+s}^{(M+1)}$.

We take (5.27) as inductive definition of $h_{i}^{N}$. A simple calculation shows that the solution of (5.27) is

$$
h_{i}^{N}=\frac{\pi}{2}\left[1-2^{-N} \sum_{t=0}^{i-1}\left(\begin{array}{c}
N \\
t
\end{array}\right)\right]=\varrho(i, N)
$$

From (5.26) we now conclude that in particular all the points

$$
w^{N}(n-1,1)=\left(0, h_{1}^{N}, \ldots h_{n-1}^{N}\right)
$$

are in $d_{n}^{(N)}$. This ends the proof of Lemma 5.2. 
For later purposes we remark that $\varrho(i, N) \geqq \frac{\pi}{2}\left(1-2^{-N / 2} \gamma_{i}\right)$, where

$$
\gamma_{i}=\max _{N \geqq 1} 2^{-N / 2} \sum_{t=0}^{i-1}\left(\begin{array}{c}
N \\
t
\end{array}\right) \text {. }
$$

Hence (b) of Lemma (5.2) implies the following corollary.

Corollary 5.3. $C_{k}^{(N)}$ contains the set

$$
\left\{\underline{\zeta}|| \arg \zeta_{i} \mid<\max \left\{0, \frac{\pi}{2}\left(1-2^{-N / 2} \gamma_{i}\right)\right\}, 1 \leqq i \leqq k\right\} .
$$

\section{The Temperedness Estimate}

In this chapter we derive the estimates (4.6) for the analytic continuation $S_{k}\left(\zeta^{0} \mid \underline{\xi}\right)$ of the Euclidean Green's functions. It is at this stage only that we have to use the linear growth condition. We point out that using $E 0^{\prime \prime}$ instead of $E 0^{\prime}$ would simplify and shorten our argument considerably.

In a first step we derive from $\left(E 0^{\prime}\right)$ the temperedness estimate (4.5) on the real analytic functions $S_{k}(\underline{\xi})$. This is the most complicated part of this chapter and it will be discussed in Section VI.1. The estimate (4.6) for the analytically continued functions $S_{k}\left(\underline{\zeta^{0}} \mid \underline{\xi}\right)$ will be proven by induction in Section VI.2.

\section{VI.1. From Distributions to Functions}

At a first glance it might look rather trivial to derive an estimate of the type (4.5) from $E 0^{\prime}$ and from the fact that $S_{k}(f)$ is given by the ordinary Riemann integral $\int S_{k}(\underline{\xi}) f(\underline{\xi}) d \xi$, with $S_{k}(\underline{\xi}) \in C^{\infty}$, say. The following example however illustrates that more detailed information about $S_{k}(\underline{\xi})$ must be available for (4.5) to be true: Let $T(x)$ be a positive $C^{\infty}$ approximation of the function $\stackrel{0}{T}(x)$ that equals $e^{x}$ for $x \in\left[n, n+e^{-2 n}\right], n \in \mathbb{Z}_{+}$, and that is 0 otherwise. Then $\left|\int T(x) f(x) d x\right|$ $\leqq \sup _{x}|f(x)|$ for all $f \in \mathscr{S}(\mathbb{R})$, but $T(x)$ is not polynomially bounded.

In Section V.1 we constructed the function $S_{k}(\underline{\xi}+\underline{\zeta})$, analytic in the polydisc $\Gamma(\underline{\xi})=\left\{\underline{\zeta}|| \zeta_{i}^{\mu} \mid<\varrho\right\}$, where $\varrho$ was a function of $\underline{\xi} \in \mathbb{R}_{+}^{\frac{4}{4 k}}$, see (5.9), Lemma 5.1. By the mean value theorem for harmonic functions (see e.g. Stein, Weiss [18])

$$
S_{k}(\underline{\xi}+\underline{\zeta})=\left|\Omega_{7}\right|^{-k} \int_{\Omega_{7}} S_{k}(\underline{\xi}+\underline{\zeta}+\underline{r} \underline{z}) d \Omega(\underline{z})
$$

where $\Omega_{7}$ denotes the surface of the unit sphere in $\mathbb{C}^{4}, \Omega_{7}=\left\{\left.z \in \mathbb{C}^{4}|| z\right|^{2}\right.$ $\left.=\sum_{\mu=1}^{4}\left|z^{\mu}\right|^{2}=1\right\}$ and $\left|\Omega_{7}\right|=\frac{2}{4 !} \pi^{4}$ is its surface area; $d \Omega(z)=\prod_{i=1}^{k} d \Omega\left(z_{i}\right)$, where $d \Omega\left(z_{i}\right)$ is the element of surface area on $\Omega_{7}$. Furthermore $\underline{r} \underline{z}=\left(r_{1} z_{1}, \ldots, r_{k} z_{k}\right)$ with $r_{i}>0$ such that $\left|\zeta_{i}\right|+r_{i}<\varrho$. Here and in the following $\underline{\xi}$ and $\varrho=\varrho(\underline{\xi})$ take fixed values. Notice that $\varrho$ is always less than 1 . Let now $h(\cdot)$ be a positive $C^{\infty}$ function with support in $\left[\frac{1}{2}, 1\right]$ such that for some $c>0, p>1$

$$
|h|_{n} \leqq c(n !)^{p} \quad \text { and } \int h(r) r^{7} d r=1 .
$$


Such a function $h$ exists (for any $p>1$ ) by the theorem of Carleman-MandelbrojtOstrowski, see e.g. Mandelbrojt [10] (take e.g. $k(x)=$ const. $\exp \left[-\left(x-\frac{1}{2}\right)^{-\beta}\right]$ $\cdot \exp \left[-(1-x)^{-\beta}\right]$ with $\left.\beta>\frac{1}{p-1}\right)$. We now set for $z \in \mathbb{C}^{4}$

and

$$
\begin{aligned}
g_{\varrho}(z) & =\left|\Omega_{7}\right|^{-\frac{1}{2}} \cdot\left(8 \varrho^{-1}\right)^{8} h\left(8 \varrho^{-1}|z|\right) \\
k_{\varrho}(z) & =\int g_{\varrho}\left(z-z^{\prime}\right) g_{\varrho}\left(z^{\prime}\right) d^{8} z^{\prime} \\
k_{\varrho}\left(z, y^{\prime}\right) & =\int g_{\varrho}\left(z-z^{\prime}\right) g_{\varrho}\left(z^{\prime}\right) d^{4} x^{\prime},
\end{aligned}
$$

where $d^{8} z^{\prime} \equiv d^{4} x^{\prime} d^{4} y^{\prime}, z^{\prime}=x^{\prime}+i y^{\prime}$. Hence

and

$$
\text { supp } k_{\varrho} \in\left\{z \in \mathbb{C}^{4}|| z \mid<\varrho / 4\right\} \text {, }
$$

$$
\int k_{\varrho}(z) d^{8} z=1 .
$$

Notice that $k_{e}$ is a function of $|z|$ only. We therefore may combine (6.1) and (6.3) to obtain with $k_{e}(z)=\prod_{i} k_{e}\left(z_{i}\right)$,

$$
S_{k}(\underline{\xi}+\underline{\zeta})=\int S_{k}(\underline{\xi}+\underline{\zeta}+\underline{z}) k_{\varrho}(\underline{z}) d^{8 k} z
$$

whenever $\left|\zeta_{i}\right|<\varrho / 2, i=1, \ldots, k$. By Fubini's theorem the order of integration on the right hand side of (6.4) is arbitrary. We thus may define

$$
T_{k}(\underline{\xi}+\underline{\zeta})=\int S_{k}(\underline{\xi}+\underline{\zeta}+\underline{x}) k_{\varrho}\left(\underline{x}+i \underline{y}, \underline{y}^{\prime}\right) d^{4 k} x
$$

such that (6.4) becomes

$$
S_{k}(\underline{\xi}+\underline{\zeta})=\int T_{k}(\underline{\xi}+\underline{\zeta}+i \underline{y}) d^{4 k} y d^{4 k} y^{\prime} .
$$

(Notice that $T_{k}$ depends on $y$ and $\underline{y}^{\prime}$ also via $k_{e}$.) Finally taking into account the support properties of $k_{e}$ and of $g_{e}$ we find that the integration in (6.6) goes only over the region where $\left|y_{i}\right|<\varrho / 4,\left|y_{i}^{\prime}\right|<\varrho / 8$ and therefore

$$
\left|S_{k}(\underline{\xi})\right| \leqq \sup _{\substack{y_{i}|<e / 4\\| y_{i} \mid<e / 8}}\left|T_{k}(\underline{\xi}+i \underline{y})\right| .
$$

The remainder of this section is devoted to the derivation of a bound on $T_{k}$ which combined with (6.7) gives the temperedness estimate (4.5).

The main idea is simple. By $(6.5), T_{k}(\xi)$ is a regularization of (the distribution) $S_{k}$. Just as $S_{k}, T_{k}$ satisfies some positivity property; in other words, $T_{k}(\underline{\xi})$ can be written as the scalar product $\left(\Psi_{1}, \Psi_{2}\right)$ of two vectors in $\mathscr{H}$. Then $\left(\Psi_{1}, e^{-z H} \Psi_{2}\right)$ defines an analytic continuation of $T_{k}(\underline{\xi})$ in one variable (as in Section V.1), whose absolute value is bounded by $\left\|\Psi_{1}\right\| \cdot\left\|\Psi_{2}\right\|$. Bounds on $\left\|\Psi_{i}\right\|$ follow from $E 0^{\prime}$. Repeating this procedure $4 k$ times we obtain analytic continuations of $T_{k}(\xi)$ in $4 k$ linearly independent directions. Analytic completion then leads to the function $T_{k}(\underline{\xi}+\zeta)$, the modulus of which we can estimate by using the maximum principle. This will give the bound (4.5) on $S_{k}(\xi)$ by (6.7).

For given $\underline{\xi} \in \mathbb{R}_{+}^{4 k}$ we define $\underline{\xi}, \gamma, \mathscr{R}_{\mu} \in \mathrm{SO}_{4}$ and the vectors $e_{1}, \ldots e_{4}$ as in (5.6), $(5.10 / 11)$. We use $g_{e}\left(\mathscr{R}_{\mu} x+i y\right)=g_{e}(x+i y) ; k_{\varrho}\left(\mathscr{R}_{\mu} x+i y, y^{\prime}\right)=k_{\varrho}\left(x+i y, y^{\prime}\right)$ and 
we simply write $g_{\varrho}(x)$ and $k_{\varrho}(x)$ for $g_{\varrho}(x+i y)$ and $k_{\varrho}\left(x+i y, y^{\prime}\right)$ respectively. Then using Euclidean covariance and (6.5) we find for all $u_{i}^{v} \geqq 0$, and $1 \leqq n \leqq k, 1 \leqq \mu \leqq 4$,

$$
\begin{aligned}
& T_{k}(\underline{0}+\underline{u} e)=T_{k}\left(\mathscr{R}_{\mu} \underline{\underline{\xi}}+\underline{u} \mathscr{R}_{\mu} e\right) \\
& =\int S_{k}(\underline{\xi}) k_{\varrho}\left(\underline{\xi}-\mathscr{R}_{\mu} \underline{\underline{\xi}}-\underline{u} \mathscr{R}_{\mu} e\right) d^{4 k} \xi
\end{aligned}
$$

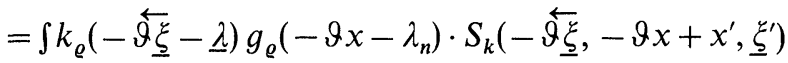

$$
\begin{aligned}
& \cdot g_{g}\left(x^{\prime}\right) k_{\varrho}\left(\underline{\xi}^{\prime}-\underline{\lambda}^{\prime}\right) d^{4(n-1)} \xi d^{4(k-n)} \xi^{\prime} d^{4} x d^{4} x^{\prime} \text {. }
\end{aligned}
$$

Here $\underline{\lambda}=\left(\lambda_{1}, \ldots \lambda_{n-1}\right)$ and $\underline{\lambda}^{\prime}=\left(\lambda_{n+1}, \ldots \lambda_{k}\right)$, where $\lambda_{i}=\mathscr{R}_{\mu} \xi_{i}+u_{i} \mathscr{R}_{\mu} e \in \mathbb{R}^{4}$ and $1 \leqq n \leqq k$. For later purposes we remark that

$$
\begin{aligned}
\left(\lambda_{i}\right)^{2} & =\left(\stackrel{0}{\xi}_{i}+\sum_{\mu} u_{i}^{\mu} e_{\mu}\right)^{2} \\
& \leqq 2 \stackrel{0}{\xi}_{i}^{2}+2\left(4 \operatorname{ctg}^{2} \gamma+3\right)\left(\sum_{\mu} u_{i}^{\mu}\right)^{2}, \quad \text { by }(5.11) \\
& \leqq \text { const. } \varrho^{-2}\left(\stackrel{0}{\xi}_{i}^{2}+\left(\sum_{\mu} u_{i}^{\mu}\right)^{2}\right),
\end{aligned}
$$

as $\operatorname{ctg} \gamma \leqq 2(1+\operatorname{ctg} 2 \gamma) \leqq 2 c \varrho^{-1}$ by (5.9) and (5.10).

Equation (6.8) exhibits $T_{\varrho}(\underline{\underline{\xi}}+\underline{u} e)$ as the scalar product $\left(\Psi_{1}, \Psi_{2}\right)$ of the two vectors

and

$$
\Psi_{1}=\int \Psi_{n}(x, \underline{\xi}) g_{\varrho}\left(-\vartheta x-\lambda_{n}\right) \prod_{i=1}^{n-1} k_{\varrho}\left(-\vartheta \xi_{n-i}-\lambda_{i}\right) d x d \xi
$$

$$
\Psi_{2}=\int \Psi_{k-n+1}\left(x^{\prime}, \underline{\xi}^{\prime}\right) g_{\varrho}\left(x^{\prime}\right) \prod_{i=1}^{k-n} k_{\varrho}\left(\xi_{i}^{\prime}-\lambda_{n+i}\right) d x^{\prime} d \xi^{\prime}
$$

As in Section V.1 we find the analytic continuation of $T_{k}$ in the variable $u_{n}^{\mu}$ by sandwiching $e^{-i v_{n}^{\mu} H}$ between $\Psi_{1}$ and $\Psi_{2}$ : for $\underline{w} \in \mathscr{P}_{n \mu}$, where

$$
\begin{gathered}
\mathscr{P}_{n \mu} \equiv\left\{\underline{w} \in \mathbb{C}^{4 k}|| \arg w_{n}^{\mu} \mid<\pi / 2, \arg w_{i}^{v}=0 \text { for } i \neq n \text { or/and } v \neq \mu\right\}, \\
T_{k}(\underline{\underline{\xi}}+\underline{w} e)=\left(\Psi_{1}, e^{-i v_{n}^{\mu} H} \Psi_{2}\right), \text { and } \\
\left|T_{k}(\underline{0}+\underline{w} e)\right| \leqq\left\|\Psi_{1}\right\| \cdot\left\|\Psi_{2}\right\|, \text { uniformly in } v_{n}^{\mu} .
\end{gathered}
$$

We defer the proof that by $E 0^{\prime}$ for some sequence $\sigma_{n}$ of factorial growth,

$$
\left\|\Psi_{1}\right\| \leqq \sigma_{n} \varrho^{-n(s+8)}\left(1+\sum_{i=1}^{n} \lambda_{i}^{2}\right)^{n s / 2}
$$

and correspondingly for $\left\|\Psi_{2}\right\|$ (with $n$ being replaced by $k-n+1$ and $\sum_{i=1}^{n} \lambda_{i}^{2}$ by $\left.\sum_{i=n+1}^{k} \lambda_{i}^{2}\right)$. Combining (6.9) and (6.12) with (6.11) we find for $\underline{w} \in \mathscr{P}_{n \mu}$,

$$
\left|T_{k}(\underline{0} \underline{\xi}+\underline{w} e)\right| \leqq \sigma_{k}^{\prime} \varrho^{-2 k(s+8)}\left(1+\sum_{i} \stackrel{\xi}{\xi}_{i}^{2}\right)^{k s / 2}\left|1+\sum_{i, v} w_{i}^{v}\right|^{k s},
$$


where $\sigma_{k}^{\prime}$ is again of factorial growth. This bound holds uniformly in the parameters $y, y^{\prime}$ on which $T_{k}$ also depends. In order to get the last factor in the expression on the r.h.s. of (6.13) we have used that $1+\Sigma u_{i}^{v} \leqq\left|1+\Sigma w_{i}^{v}\right|$. We now may study the functions

$$
R_{k}^{n \mu}(\underline{r}) \equiv\left(1+\Sigma w_{i}^{v}\right)^{-k s} T_{k}(\underline{q}+\underline{w} e),
$$

$r_{i}^{v}=\ln w_{i}^{v}$, which is analytic in the tube $\mathscr{T}_{n \mu}=\left\{\underline{r}|| \operatorname{Im} r_{n}^{\mu} \mid<\pi / 2, \operatorname{Im} r_{i}^{v}=0\right.$ for $i \neq n$ or/and $v \neq \mu\}$ and whose modulus is uniformly bounded there by

$$
\sigma_{k}^{\prime} \varrho^{-2 k(s+8)}\left(1+\sum_{i}^{0} \xi_{i}^{2}\right)^{k s / 2} .
$$

For $\operatorname{Im} \underline{r}=0$, the functions $R_{k}^{n \mu}(\underline{r})$ are all equal, independent of $n, \mu$ [namely, equal to $\left.\left(1+\Sigma u_{i}^{v}\right)^{-k s} T_{k}(\underline{\underline{\xi}}+\underline{u} e)\right]$. Hence the Malgrange-Zerner theorem applies and there is a function $R_{k}(\underline{r})$, analytic in the convex envelope $\mathscr{T}$ of $\mathscr{T}=\bigcup_{n \mu} \mathscr{T}_{n \mu}$, which continues all the $R_{k}^{n \mu}(\underline{r})$. We claim that $\left|R_{k}(\underline{r})\right|$ is again bounded by $(6.14)$ for all $\underline{r}$ in $\mathscr{T}$. For a proof let us assume $R_{k}(\underline{r})$ takes a value $A$ at a point $\underline{\underline{0}} \in \mathbb{T} \sim \mathscr{T}$, which it does not take in $\mathscr{T}$. Then $\left(R_{k}(\underline{r})-A\right)^{-1}$ is analytic in an open neighborhood $\hat{\mathscr{T}} \subset \mathscr{T}$ of $\mathscr{T}$ but not in all of $\mathscr{T}$. This is impossible, because $\mathscr{T}$ is the envelope of holomorphy of $\hat{\mathscr{T}}$. Hence

$$
\sup _{\underline{r} \in \mathscr{T}}\left|R_{k}(r)\right|=\sup _{\underline{r} \in \mathscr{T}}\left|R_{k}(\underline{r})\right|
$$

and the assertion follows. The function

$$
T_{k}(\underline{0}+\underline{w} e) \equiv\left(1+\Sigma w_{i}^{v}\right)^{k s} R_{k}(\ln \underline{w})
$$

analytically continues $T_{k}(\stackrel{0}{\xi}+\underline{u} e)$ to the domain $\mathscr{D}=\left\{\underline{w}\left|\sum_{i, v}\right| \arg w_{i}^{v} \mid<\pi / 2\right\}$ and it satisfies the bound (6.13) for all $\underline{w} \in \mathscr{D}$.

Now we go back to (6.7) and use (5.12): $\underline{\xi}+i \underline{y}=\underline{\xi}+\underline{w} e$, where

$$
w_{i}^{\mu}=2^{-4} \xi_{i}^{0} \operatorname{tg} \gamma+i\left(2^{-3} \operatorname{tg} \gamma y_{i}^{0}+2^{-2} \sum_{r=1}^{3} \sigma_{r \mu} y_{i}^{r}\right)
$$

and, as $\operatorname{tg} \gamma \leqq 1,\left|y_{i}^{0}\right|<\varrho / 4,\left|y_{i}^{r}\right|<\varrho / 4$,

$$
\left|w_{i}^{\mu}\right| \leqq \frac{1}{4}\left(\xi_{i}^{0}+\varrho\right) .
$$

Also note that $\left(\stackrel{\xi}{\xi}_{i}\right)^{2}=\left(\frac{1}{2} \xi_{i}^{0}\right)^{2}+\vec{\xi}_{i}^{2}<\left(\xi_{i}\right)^{2}$. Hence from (6.13), (6.7), and (5.9) we get

$$
\begin{aligned}
\left|S_{k}(\xi)\right| & \leqq \sigma_{k}^{\prime} \varrho^{-2 k(s+8)}\left(1+\sum_{i}\left(\xi_{i}\right)^{2}\right)^{k s / 2}\left(1+\sum_{i} \xi_{i}^{0}+k \varrho\right)^{k s} \\
& \leqq \tau_{k}\left[\left(1+\max _{1 \leqq j \leqq k} \mid \vec{\xi}_{j}\right)\left(1+\sum_{1}^{k} \xi_{j}^{0}\right)\left(1+\sum_{j}^{k}\left(\xi_{j}^{0}\right)^{-1}\right)\right]^{k t}
\end{aligned}
$$

for some sequence $\tau_{k}$ of factorial growth and $t=5(s+8)$. Inequality (6.16) is the temperedness estimate (4.5). 
It remains to prove the bound (6.12) on $\left\|\Psi_{1}\right\|$ and $\left\|\Psi_{2}\right\|$ respectively. From (6.10) and (5.2) we get

$$
\begin{aligned}
& \left\|\Psi_{1}\right\|^{2}=\int g_{\varrho}\left(-\vartheta x-\lambda_{n}\right) \prod_{i=1}^{n-1} k_{\varrho}\left(-\vartheta \xi_{i}-\lambda_{i}\right) S_{2 n-1}\left(-\overleftarrow{\vartheta} \underline{\xi},-\vartheta x+x^{\prime}, \underline{\xi}^{\prime}\right) \\
& \cdot g_{\varrho}\left(-\vartheta x^{\prime}-\lambda_{n}\right) \prod_{i=1}^{n-1} k_{\varrho}\left(-\vartheta \xi_{n-i}^{\prime}-\lambda_{i}\right) d x d x^{\prime} d \xi d \xi^{\prime} \\
& =\int g_{\varrho}\left(-x_{n}-\lambda_{n}\right) \prod_{i=1}^{n-1} k_{\varrho}\left(x_{i+1}-x_{i}-\lambda_{i}\right) \Im_{2 n}\left(x_{1}, \ldots x_{n}, y_{n}, \ldots y_{1}\right) \\
& \cdot g_{\varrho}\left(-\vartheta y_{n}-\lambda_{n}\right) \prod_{i=1}^{n-1} k_{\varrho}\left(-\vartheta\left(y_{i}-y_{i+1}\right)-\lambda_{i}\right) d x d y \\
& \leqq \sigma_{2 n} \cdot \sup _{\substack{x, y \\
|\underline{\alpha}|+\mid \underline{\underline{y} \mid \leqq 2 n s}}} \mid\left(1+\sum_{i} x_{i}^{2}+\sum_{i} y_{i}^{2}\right)^{n s} D_{x} \underline{\alpha} g_{\varrho}\left(x_{n}-\lambda_{n}\right) \prod_{i=1}^{n-1} k_{\varrho}\left(x_{i}-x_{i+1}-\lambda_{i}\right) \\
& \cdot D_{y} \underline{\underline{\beta}} g_{\varrho}\left(y_{n}-\lambda_{n}\right) \prod_{i=1}^{n-1} k_{\varrho}\left(y_{i}-y_{i+1}-\lambda_{i}\right) \mid \text {. }
\end{aligned}
$$

By (6.3), the function under the sup in (6.17) is nonzero only if

$$
\left|x_{n}-\lambda_{n}\right|<\varrho / 8,\left|x_{i}-x_{i+1}-\lambda_{i}\right|<\varrho / 4 \text { for } i=1, \ldots n-1
$$

and similarly for $y_{i}$. This implies that $\left|x_{n}\right|<\left|\lambda_{n}\right|+\varrho / 8,\left|x_{i}\right|<\sum_{j=1}^{n}\left|\lambda_{j}\right|+(n-1) \varrho / 4$ and $[$ as $(n-1) \varrho / 4$ is always smaller than 1$]$

$$
1+\sum_{i=1}^{n} x_{i}^{2}+\sum_{i=1}^{n} y_{i}^{2} \leqq 8 n^{2}\left(1+\sum_{i=1}^{n} \lambda_{i}^{2}\right)
$$

Furthermore by $(6.2 / 3)$, for some $c>0$

$$
\sup \left|D^{\alpha} g_{\varrho}\right|+\sup \left|D^{\alpha} k_{\varrho}\right|<c(|\alpha| !)^{p}\left(8 \varrho^{-1}\right)^{|\alpha|+8} .
$$

This together with $(6.17 / 18)$ yields $(6.12)$.

This completes the proof of the temperedness estimate (4.5).

\section{VI.2. Continuing the Estimates}

In this section we prove the temperedness estimates $\left(T E_{k}\right)$ for $k \in \mathbb{Z}_{+}$. In essence we will repeat the arguments of Section V.2 but carrying along the estimates on the analytic functions $S_{k}\left(\underline{\zeta}^{0} \mid \vec{\xi}\right)$. Our main tool will be the maximum principle, see $(6: 15)$. In order to dispose of the spatial variables $\underline{\xi}$ we let $\mathscr{B}_{p, n}$ be the Banach space of all continuous functions on $\mathbb{R}^{3 n}$, satisfying

$$
\|f\|_{p}=\sup _{\vec{\xi} \in \mathbb{R}^{3 n}}\left|\left(1+\max _{1 \leqq i \leqq n}\left|\vec{\xi}_{i}\right|\right)^{-p} f(\vec{\xi})\right|<\infty
$$

Then $S_{k}\left(\underline{\xi}^{0} \mid \cdot\right)$ are real analytic functions in the $\underline{\xi}^{0}$ variables, for $\xi_{i}^{0}>0$, with values in $\mathscr{B}_{k t, k}$. By (4.5)

$$
\left\|S_{k}\left(\underline{\xi}^{0} \mid \cdot\right)\right\|_{k t} \leqq \alpha k^{\beta k}\left[\left(\frac{1+\Sigma \xi_{j}^{0}}{k}\right)\left(\frac{1+\Sigma\left(\xi_{j}^{0}\right)^{-1}}{k}\right)\right]^{k t}
$$


for some constants $\alpha>0 ; \beta>0$. We write $S_{k}\left(\underline{\xi}^{0} \mid \cdot\right)$ simply as $S_{k}\left(\underline{\xi}^{0}\right)$ or as $S_{k}(\underline{\xi})$ - thus suppressing the superscript 0 .

Suppose now we have already shown that $\left.S_{k}(\zeta) \cdot\right) \equiv S_{k}(\zeta)$ defines a real analytic function from $C_{k}^{(N)}$ to $\mathscr{B}_{k t, k}$. Then we define

$$
S_{k, \varepsilon}(\zeta)=\left(\frac{1+\Sigma\left(\zeta_{j}+\varepsilon\right)}{k}\right)^{-k t}\left(k^{-1}+\varepsilon^{-1}\right)^{-k t} S_{k}(\underline{\zeta}+\underline{\varepsilon}),
$$

where $\underline{\varepsilon}$ is the vector $(\varepsilon, \ldots \varepsilon), \varepsilon>0$. Now let $k=n+m-1,(x, \underline{\zeta}) \in D_{n}^{(N)} ;\left(x, \underline{\zeta}^{\prime}\right) \in D_{m}^{(N)}$ and $z=x+i y \in \mathbb{C}_{+}$. Then the Schwarz inequality

$$
\left\|S_{k}\left(\underline{\bar{\zeta}}, 2 x, \underline{\zeta}^{\prime}\right)\right\|_{k t} \leqq\left(\left\|S_{2 n-1}(\underline{\bar{\zeta}}, 2 x, \underline{\zeta})\right\|_{(2 n-1) t} \cdot\left\|S_{2 m-1}\left(\underline{\bar{\zeta}}^{\prime}, 2 x, \underline{\zeta}^{\prime}\right)\right\|_{(2 m-1) t}\right)^{\frac{1}{2}}
$$

is an immediate consequence of $P_{N}$, Eq. (5.17), and of the definition (6.19) of the norms $\|\cdot\|_{p}$. Inequality (6.22) holds for $S_{,, \varepsilon}$ as well: For $k=n+m-1$,

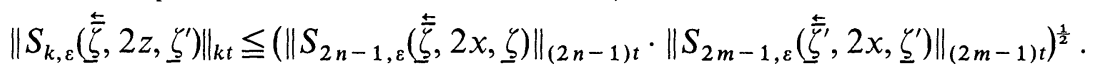

To get (6.23) from (6.22), we only have to prove that for $\operatorname{Re} \zeta_{i}>0, \operatorname{Re} \zeta_{i}^{\prime}>0, x>0$,

$$
\begin{aligned}
& \left|k^{-1}\left(1+\sum_{1}^{n-1} \bar{\zeta}_{i}+2 z+\sum_{1}^{m-1} \zeta_{i}^{\prime}\right)\right|^{2 k} \\
& \geqq\left[(2 n-1)^{-1}\left(1+2 \sum_{1}^{n-1} \operatorname{Re} \zeta_{i}+2 x\right)\right]^{2 n-1}\left[(2 m-1)^{-1}\left(1+2 \sum_{1}^{m-1} \operatorname{Re} \zeta_{i}^{\prime}+2 x\right)\right]^{2 m-1}
\end{aligned}
$$

and

$$
\left(k^{-1}+\varepsilon^{-1}\right)^{2 k} \geqq\left[(2 n-1)^{-1}+\varepsilon^{-1}\right]^{2 n-1}\left[(2 m-1)^{-1}+\varepsilon^{-1}\right]^{2 m-1} .
$$

Clearly (6.24) follows if we can show that

$$
\left.\left|k^{-1}\left(1+\sum_{1}^{n-1} \operatorname{Re} \zeta_{i}+2 x+\sum_{1}^{m-1} \operatorname{Re} \zeta_{i}^{\prime}\right)\right|\right|^{2 k} \geqq \text { r.h.s. of (6.24). }
$$

Both inequalities (6.25) and (6.26) can be brought into the form

$$
\left(\frac{A+B}{r+s}\right)^{r+s} \geqq\left(\frac{A}{r}\right)^{r}\left(\frac{B}{s}\right)^{s}
$$

for $A$ and $B$ both positive; (6.27) follows from the convexity of the function $f(x)=\ln x$.

We now claim that for $\underline{\zeta} \in C_{k}^{(N)}$,

$$
\left\|S_{k, \varepsilon}(\zeta)\right\|_{k t} \leqq \alpha k^{\beta k} \cdot 2^{\beta k N}
$$

with $\alpha$ and $\beta$ as in (6.20). We prove (6.28) by induction. For $N=0,(6.28)$ follows from inequality (6.20). Now assume we have verified inequality (6.28) for $N=0,1, \ldots M$ and for all $k \in \mathbb{Z}_{+}$, all $\varepsilon>0$. Then for $(x, \zeta) \in D_{n}^{(M)},\left(x, \underline{\zeta}^{\prime}\right) \in D_{m}^{(M)}, z=x+i y \in \mathbb{C}_{+}$, $k=n+m-1$, we have by (6.23) and the induction assumption

$$
\begin{aligned}
& \left\|S_{k, \varepsilon}\left(\zeta, 2 z, \zeta^{\prime}\right)\right\|_{k t} \\
& \quad \leqq\left[\alpha(2 n-1)^{\beta(2 n-1)} 2^{\beta(2 n-1) M} \cdot \alpha(2 m-1)^{\beta(2 m-1)} 2^{\beta(2 m-1) M}\right]^{\frac{1}{2}} \\
& \quad \leqq \alpha k^{\beta k} \cdot 2^{\beta k(M+1)} .
\end{aligned}
$$


As $C_{k}^{(M+1)}$ is just the envelope of holomorphy of the region

$$
\hat{C}_{k}^{(M+1)}=\bigcup_{n+m-1=k}\left\{\left(\underline{\underline{\zeta}}, 2 z, \underline{\zeta}^{\prime}\right) \mid(x, \underline{\zeta}) \in D_{n}^{(M)},\left(x, \underline{\zeta}^{\prime}\right) \in D_{m}^{(M)}, z=x+i y \in \mathbb{C}_{+}\right\},
$$

we can use the maximum principle (see (6.15) and e.g. Vladimirov [19] p. 178), to conclude that (6.28) holds for $N=M+1$. We argue first point-wise, i.e. for fixed values of the spatial variables $\underline{\xi}$ and then take the norms $\|\cdot\|_{k t}$.

Our final step will be to eliminate $N$ from the right hand side of (6.28). (Notice that the right hand side of (4.6) does not depend on $N$ either!) For a given $\zeta \in \mathbb{C}_{+}^{k}$ we want to find an $N=N(\underline{\zeta})$ such that $\underline{\zeta} \in C_{k}^{(N)}$. Choose $s$ such that $\left|\arg \zeta_{r}\right| \leqq\left|\arg \zeta_{s}\right|$ for all $1 \leqq r \leqq k$. Now

$$
\begin{aligned}
& \left|\arg \zeta_{s}\right|+\arcsin \frac{\operatorname{Re} \zeta_{s}}{\left|\zeta_{s}\right|}=\pi / 2, \text { hence } \\
& \left|\arg \zeta_{s}\right| \leqq \frac{\pi}{2}\left(1-\frac{\operatorname{Re} \zeta_{s}}{\left|\zeta_{s}\right|} \cdot \frac{2}{\pi}\right) .
\end{aligned}
$$

Choose the integer $N=N(\zeta)$ such that [with $\gamma_{k}$ as in (5.28)]

$$
2^{-N / 2} \gamma_{k} \leqq \frac{\operatorname{Re} \zeta_{s}}{\left|\zeta_{s}\right|} \cdot \frac{2}{\pi} \leqq 2^{-(N-1) / 2} \gamma_{k}
$$

Then by Corollary 5.3, $\zeta \in C_{k}^{(N)}$. Inserting (6.30) in (6.28) shows that

$$
\begin{aligned}
\left\|S_{k, \varepsilon}\left(\zeta^{0}\right)\right\|_{k t} & \leqq \alpha k^{\beta k}\left[\frac{\gamma_{k} \cdot \pi}{\sqrt{2}} \frac{\left|\zeta_{s}^{0}\right|}{\operatorname{Re} \zeta_{s}^{0}}\right]^{2 \beta k} \\
& \leqq \alpha k^{\beta k}\left[\frac{\gamma_{k} \cdot \pi}{\sqrt{2}}\right]^{2 \beta k} \cdot\left[\left(1+\Sigma\left|\zeta_{i}^{0}\right|\right)\left(1+\Sigma\left(\operatorname{Re} \zeta_{i}^{0}\right)^{-1}\right)\right]^{2 \beta k}
\end{aligned}
$$

We combine this with (6.21), choose $\varepsilon=\frac{1}{2} \min _{i} \operatorname{Re} \zeta_{i}^{0}$ and "undo" the norm $\|\cdot\|_{k t}$ to get for $\underline{\zeta}^{0} \in \mathbb{C}_{+}^{k}, \underline{\vec{\xi}} \in \mathbb{R}^{3 k}$,

and

$$
\left\|S_{k}\left(\zeta^{0}\right)\right\|_{k t} \leqq\left|\frac{1+\Sigma \zeta_{i}^{0}}{k}\right|^{k t}\left(k^{-1}+\varepsilon^{-1}\right)^{k t}\left\|S_{k, \varepsilon}\left(\zeta^{0}-\underline{\varepsilon}\right)\right\|_{k t}
$$

$$
\left|S_{k}\left(\zeta^{0} \mid \underline{\xi}\right)\right| \leqq c_{k}\left(1+\max _{i}\left|\vec{\xi}_{i}\right|\right){ }^{k t} \cdot\left[\left(1+\Sigma\left|\zeta_{i}^{0}\right|\right)\left(1+\Sigma\left(\operatorname{Re} \xi_{i}^{0}\right)^{-1}\right)\right]^{(2 \beta+t) k}
$$

where $c_{k}=\alpha k^{(\beta-t) k}\left(\gamma_{k} \pi\right)^{2 \beta k} 2^{(t-\beta) k}$. From (5.28) one easily gets $\gamma_{k}<c^{k}$ for some $c>1$, and hence $c_{k}<a b^{k^{2}}$ for some constants $a, b>0$. Inequality (6.31) is the desired temperedness estimate (4.6) with $t^{\prime}=2 \beta+t$.

\section{Appendix (by Stephen Summers)}

Theorem. Condition E0" implies E0'.

Proof. We prove the equivalent statement that for $T \in \mathscr{S}^{\prime}\left(\mathbb{R}^{n}\right)$ with

$$
\left|T\left(f_{1} \times f_{2} \times \ldots f_{n}\right)\right| \leqq \prod_{k=1}^{n}\left|f_{k}\right|_{r}
$$


for all $f_{i} \in \mathscr{S}(\mathbb{R})$ and some fixed $r$, it follows that

$$
|T(g)| \leqq c^{n}|g|_{n \cdot t}
$$

for all $g \in \mathscr{S}\left(\mathbb{R}^{n}\right)$, some fixed constant $c$ not depending on $n$ or $g$ and $t=2 r+7$.

We use a Hermite expansion for $T$, see Schwartz [14]. (Hermite expansion can also be used to prove the nuclear theorem, Simon [17].) Writing $H_{i}$ for $H_{i_{1}} \otimes H_{i_{2}} \ldots \otimes H_{i_{n}}$, where $H_{i}$ is the $i$-th Hermite function, we get

$$
T(g)=\sum_{\underline{i}} \tau_{\underline{i}} \gamma_{\underline{i}}
$$

where $\underline{i}=\left(i_{1}, \ldots i_{n}\right), i_{k} \in \mathbb{Z}_{+}$, and $\tau_{\underline{i}}=T\left(H_{\underline{i}}\right), \quad \gamma_{\underline{i}}=\int H_{\underline{i}}(x) g(x) d^{n} x$. We set $(1+\underline{i})$

$$
\begin{aligned}
& \equiv \prod_{k=1}^{n}\left(1+i_{k}\right) \text { and obtain } \\
& \qquad \begin{aligned}
|T(g)| & \leqq \sum_{\underline{i}}(1+\underline{i})^{-2}\left|(1+\underline{i})^{-s} \tau_{\underline{i}}\right|\left|(1+\underline{i})^{s+2} \gamma_{\underline{i}}\right| \\
& \leqq c_{1}^{n} \sup _{\underline{\underline{i}}}\left|(1+\underline{i})^{-s} \tau_{\underline{\underline{i}}}\right| \cdot \sup \left|(1+\underline{i})^{s+2} \gamma_{\underline{i}}\right|
\end{aligned}
\end{aligned}
$$

where $c_{1}^{n}=\sum_{i}(1+\underline{i})^{-2}=\left(\sum_{i=0}^{\infty}(1+i)^{-2}\right)^{n}$. To finish the proof, we have to determine $s$ such that $\overline{\mid}(1+\underline{i})^{-s} \tau_{\underline{j}} \mid$ is uniformly bounded in $\underline{i}$.

Introducing $a_{k}^{ \pm}=\frac{1}{\sqrt{2}}\left(x_{k} \mp \partial_{k}\right)$, we have $a_{k}^{+} H_{i_{k}}=\sqrt{1+i_{k}} H_{i_{k}+1}$ and $a_{k} H_{i_{k}}$ $=\sqrt{i_{k}} H_{i_{k}-1}, a_{k} a_{k}^{+} H_{i_{k}}=\left(1+i_{k}\right) H_{i_{k}}=\left(1+x_{k}^{2}-\partial_{k}^{2}\right) H_{i_{k}}$. Furthermore (dropping the index $k$ for the moment)

$$
\begin{aligned}
\left|H_{i}\right|_{r} & =\sup _{\alpha \leqq r}\left\|\left(1+x^{2}\right)^{r / 2} \partial^{\alpha} H_{i}\right\|_{\infty} \\
& \leqq c_{2} \sup _{\substack{\alpha \leqq r \\
\beta \leqq r+1}}\left\|x^{\beta} \partial^{\alpha} H_{i}\right\|_{2} \quad \text { (Sobolev inequality) } \\
& \leqq c_{3} \sup \left\|a^{ \pm} \ldots a^{ \pm} H_{i}\right\|_{2} \quad\left(\leqq 2 r+1 \text { factors } a^{+} \text {or } a^{-}\right) \\
& \leqq c_{3} \sqrt{(i+1)(i+2) \ldots(i+2 r+1)} \leqq c_{4}(1+i)^{r+1} .
\end{aligned}
$$

Here and in the following $c_{m}$ denote constants that depend on $r$ only. By (A1) and (A4),

$$
\begin{aligned}
\left|\tau_{\underline{\underline{i}}}\right| & =\left|T\left(H_{\underline{i}}\right)\right| \leqq \prod_{k}\left|H_{i_{k}}\right|_{r} \\
& \leqq c_{4}^{n}(1+\underline{i})^{r+1} .
\end{aligned}
$$

Choosing $s=r+1$, we dominate the second factor in (A3) by $c_{4}^{n}$. Finally

$$
\begin{aligned}
\left|(1+\underline{i})^{s+2} \gamma_{\underline{i}}\right| & =\left|\int\left(\prod_{k}\left(1+x_{k}^{2}-\partial_{k}^{2}\right)^{s+2} H_{i_{k}}\left(x_{k}\right)\right) g(x) d x\right| \\
& \leqq c_{5}^{n} \sup _{x \in \mathbb{R}^{4}}\left|\left(1+x^{2}\right)^{n / 2} \prod_{k=1}^{n}\left(1+x_{k}^{2}-\partial_{k}^{2}\right)^{s+2} g(x)\right| \\
& \leqq c_{6}^{n} \sup _{\substack{x \in \mathbb{R}^{n} \\
|\underline{\alpha}| \leqq 2(s+2) \cdot n}}\left|\left(1+x^{2}\right)^{n\left(\frac{1}{2}+s+2\right)} D^{\underline{\alpha}} g(x)\right| \leqq c_{6}^{n}|g|_{n t},
\end{aligned}
$$


where $t=2 s+5=2 r+7$, and

$$
c_{5}^{n}=\left|\int\left(1+x^{2}\right)^{-n / 2} H_{\underline{i}}(x) d^{n} x\right| \leqq\left\|H_{\underline{i}}\right\|_{2}\left(\int\left(1+x^{2}\right)^{-n} d^{n} x\right)^{\frac{1}{2}} .
$$

Substituting (A5) and (A6) we obtain (A2).

\title{
References
}

1. Constantinescu,F., Thalheimer, W.: Euclidean Green's functions for Jaffe fields. Commun. math. Phys. 38, 299-316 (1974)

2. Epstein, H.: Some analytic properties of scattering amplitudes in quantum field theory. In: Chretien, M., Deser, S. (Eds.): Brandeis lectures 1965, Vol. I. New York: Gordon and Breach 1966

3. Fröhlich, J.: Schwinger functions and their generating functionals. Helv. Phys. Acta 47, 265 (1974)

4. Gelfand, I. M., Shilov, G.E.: Generalized functions, Vol. II, p. 227. New York and London: Academic Press 1968

5. Glaser, V.: The positivity condition in momentum space. In: Problems of theoretical physics. Moscow: Nauka 1969

6. Glaser, V.: On the equivalence of the Euclidean and Wightman formulations of field theory. Commun. Math. Phys. 37, 257 (1974)

7. Glimm, J., Jaffe, A.: A remark on the existence of $\varphi_{4}^{4}$. Phys. Rev. Lett. 33, 440-441 (1974)

8. Glimm,J., Jaffe, A., Spencer,T.: The Wightman axioms and particle structure in the $P(\varphi)_{2}$ quantum field model. Ann. Math. 100, 585 (1974)

9. Hörmander, L.: On the division of distributions by polynomials. Arkiv Mat. 3, 555 (1958)

10. Mandelbrojt, S.: Séries adhérentes, régularisation des suites, applications. Paris: GauthierVillars 1952

11. Nelson,E.: Construction of quantum fields from Markoff fields. J. Funct. Anal. 12, 97 (1973)

12. Osterwalder, K., Schrader, R.: Axioms for Euclidean Green's functions. Commun. math. Phys. 31, 83 (1973)

13. Osterwalder, K.: Euclidean Green's functions and Wightman distributions. In: Velo, G., Wightman, A.S. (Eds.): Constructive quantum field theory, Lecture notes in physics. Berlin-HeidelbergNew York: Springer 1973

14. Schwartz, L.: Théorie des distributions, p. 260. Paris: Hermann 1966

15. Simon, B.: Positivity of the Hamiltonian semigroup and the construction of Euclidean region fields. Helv. Phys. Acta 46, 686 (1973)

16. Simon, B.: Private communication

17. Simon, B.: Distributions and their hermite expansions. J. Math. Phys. 12, 140 (1971)

18. Stein, M., Weiss, G.: Fourier analysis on Euclidean spaces, p. 38. Princeton University Press 1971

19. Velo, G., Wightman, A.S. (Eds.): Constructive quantum field theory, Lecture notes in physics. Berlin-Heidelberg-New York: Springer 1973

20. Vladimirov, V.S.: Methods of the theory of functions of several complex variables. Cambridge and London: MIT Press 1966

21. Whitney, H.: Analytic extensions of differentiable functions defined in closed sets. Trans. Amer. Math. Soc. 36, 63 (1934)

Communicated by A. S. Wightman

\author{
Konrad Osterwalder \\ Jefferson Laboratory of Physics \\ Harvard University \\ Cambridge, Mass. 02138, USA \\ Robert Schrader \\ Institut für Theoretische Physik \\ Freie Universität Berlin \\ D-1000 Berlin
}


